\title{
mTOR-Mediated Regulation of Immune Responses in Cancer and Tumor Microenvironment
}

\section{OPEN ACCESS}

Edited by:

Arvind Chhabra

Amity University Gurgaon, India

Reviewed by:

Pingping Chen,

University of Miami, United States

Alla Ivanova,

Meharry Medical College,

United States

*Correspondence:

Davoud Rostamzadeh

d.rostamzadeh@yahoo.com;

davood.rostamzadeh@yums.ac.ir

William C. Cho

williamcscho@gmail.com;

chocs@ha.org.hk

tORCID:

Davoud Rostamzadeh orcid.org/0000-0002-5528-1984

William C. Cho

orcid.org/0000-0003-4174-4586

Specialty section:

This article was submitted to

Cancer Immunity

and Immunotherapy,

a section of the journal

Frontiers in Immunology

Received: 11 September 2021 Accepted: 14 December 2021 Published: 18 February 2022

Citation:

Mafi S, Mansoori B, Taeb S, Sadeghi H, Abbasi R, Cho WC and Rostamzadeh D (2022) mTOR-

Mediated Regulation of Immune

Responses in Cancer and

Tumor Microenvironment.

Front. Immunol. 12:774103. doi: 10.3389/fimmu.2021.774103

\section{Sahar Mafi ${ }^{1,2}$, Behzad Mansoori ${ }^{3}$, Shahram Taeb ${ }^{4,5}$, Hossein Sadeghi $^{2}$, Reza Abbasi $^{2}$, William C. Cho ${ }^{6 * t}$ and Davoud Rostamzadeh ${ }^{1,2 * t}$}

${ }^{1}$ Department of Clinical Biochemistry, Yasuj University of Medical Sciences, Yasuj, Iran, ${ }^{2}$ Medicinal Plants Research Center, Yasuj University of Medical Sciences, Yasuj, Iran, ${ }^{3}$ The Wistar Institute, Molecular \& Cellular Oncogenesis Program, Philadelphia, PA, United States, ${ }^{4}$ Department of Radiology, School of Paramedical Sciences, Guilan University of Medical Sciences, Rasht, Iran, ${ }^{5}$ Medical Biotechnology Research Center, School of Paramedical Sciences, Guilan University of Medical Sciences, Rasht, Iran, ${ }^{6}$ Department of Clinical Oncology, Queen Elizabeth Hospital, Hong Kong, Hong Kong SAR, China

The mechanistic/mammalian target of rapamycin (mTOR) is a downstream mediator in the phosphatidylinositol 3-kinase (PI3K)/Akt signaling pathways, which plays a pivotal role in regulating numerous cellular functions including cell growth, proliferation, survival, and metabolism by integrating a variety of extracellular and intracellular signals in the tumor microenvironment (TME). Dysregulation of the mTOR pathway is frequently reported in many types of human tumors, and targeting the PI3K/Akt/mTOR signaling pathway has been considered an attractive potential therapeutic target in cancer. The PI3K/Akt/mTOR signaling transduction pathway is important not only in the development and progression of cancers but also for its critical regulatory role in the tumor microenvironment. Immunologically, mTOR is emerging as a key regulator of immune responses. The mTOR signaling pathway plays an essential regulatory role in the differentiation and function of both innate and adaptive immune cells. Considering the central role of $\mathrm{mTOR}$ in metabolic and translational reprogramming, it can affect tumor-associated immune cells to undergo phenotypic and functional reprogramming in TME. The mTOR-mediated inflammatory response can also promote the recruitment of immune cells to TME, resulting in exerting the anti-tumor functions or promoting cancer cell growth, progression, and metastasis. Thus, deregulated mTOR signaling in cancer can modulate the TME, thereby affecting the tumor immune microenvironment. Here, we review the current knowledge regarding the crucial role of the PI3K/Akt/mTOR pathway in controlling and shaping the immune responses in TME.

Keywords: cancer, mTOR, T cell, Tumor microenvironment, PI3K/Akt/mTOR signaling pathway, immune response

\section{INTRODUCTION}

The mammalian target of rapamycin (mTOR; now officially known as the mechanistic target of rapamycin) is a ubiquitous serine/threonine-specific protein kinase, plays a critical role in regulating numerous cellular functions, including cell growth, proliferation, survival, protein synthesis, ribosome biogenesis, autophagy, and metabolism $(1,2)$. mTOR functions within two functionally 
and structurally distinct multi-component kinase complexes called mTOR complex 1 (mTORC1) and mTOR complex 2 (mTORC2) that act as the central nodes of the phosphoinositide 3-kinase (PI3K)/Akt downstream signaling pathway (3). The activity of PI3K/Akt/mTOR pathway is frequently dysregulated in majority of human tumors and has a crucial role during tumorigenesis and cancer development (4-6). Thus, targeting the $\mathrm{PI} 3 \mathrm{~K} / \mathrm{Akt} / \mathrm{mTOR}$ signaling pathway would be an attractive potential therapeutic target in cancer (7).

The tumor microenvironment (TME) contains malignant cells and nonmalignant cells such as endothelial cells, cancer-associated fibroblasts (CAFs), and several kinds of tumor-infiltrating immune/ inflammatory cells, as well as a variety of soluble factors (cytokines and growth factors) released from cell subpopulations and plays pivotal roles in facilitating tumorigenesis, promoting tumor progression and immune evasion (8). TME is highly enriched in the immune cell populations and reciprocal signaling between immune cells and cancer cells can reduce the anti-cancer activity of endogenous tumor-infiltrating immune cells and facilitate immune evasion $(9,10)$. Studies have shown that the immune infiltration of tumors are closely associated with tumor proliferation, angiogenesis, invasion, and metastasis (11). Along with the critical role of the mTOR in cancer, recent studies have established an essential regulatory role of the mTOR in differentiation, activation, and functional properties of immune cells in which mTOR functions to coordinate and shape immune effector responses $(12,13)$. Therefore, dysregulation of this network potentially affects immune cells effector function and influences the tumor immune microenvironment (TIME) landscape in human cancers (10). As the core regulator of metabolic and translational reprogramming, mTOR is mainly involved in the central tumor immune microenvironment, affecting tumor-associated immune cells to undergo phenotypic and functional reprogramming in TME. Indeed, mTOR regulates immune responses by regulating the expression of inflammatory mediators, such as interleukin (IL)12 , IL-10, transforming growth factor (TGF- $\beta$ ), and tumor necrosis factor (TNF), as well as immune checkpoint receptors cytotoxic Tlymphocyte protein 4 (CTLA-4) and programmed death 1 (PD-1) $(12,14)$. Further, a recent study has revealed that mTOR gene expression is markedly correlated with various immune cells and immunoinhibitors in patients with clear cell renal cell carcinoma (ccRCC) (15). The mTOR-mediated inflammatory response also promotes immune cells recruitment to TME by inflammatory mediators, resulting in exerting the anti-tumor functions or augments tumor growth, progression, and metastatic capacities of cancer cells (14).

This review focuses on the current knowledge regarding the key role of the mTOR signaling pathway in controlling and shaping the immune responses in TME.

\section{mTORC1 SIGNALING PATHWAY}

mTOR is an atypical serine/threonine-specific protein kinase that belongs to the phosphatidylinositol-3 kinase-related kinases (PIKK) family (16). mTOR functions as a downstream effector of the PI3K/Akt signaling pathway in two distinct sets of intracellular complexes, mTORC1 and mTORC2 (3). Both complexes share three conserved subunits: mTOR, the catalytic subunit, DEP-containing mTOR interacting protein (DEPTOR), and mammalian lethal with SEC13 protein $8 / G$ protein $\beta$ subunit-like (mLST8/G $\beta \mathrm{L}$ ). In addition to the same subunits, the regulatory-associated protein of mTOR (RAPTOR) and proline-rich Akt substrate $40 \mathrm{kDa}$ (PRAS40) are unique subunits for mTORC1 complex, whereas rapamycin-insensitive companion of mTOR (RICTOR), protein observed with Rictor (PROTOR) and mammalian stress-activated protein kinase (SAPK)-interacting protein 1 (mSIN1) are unique components for mTORC2 complex (Figure 1) (16). The mTOR pathway senses a variety of intracellular and extracellular signals in the form of growth factors, cytokines, nutrients, and energy levels in the form of ATP, cellular stress, and inflammation. The signaling inputs downstream of these diverse signals are predominantly delivered to the PI3K/Akt pathway that eventually activates mTOR (Figure 1) (1, 12). The phosphatase and tensin homologue (PTEN), a well-characterized tumor suppressor, negatively regulates $\mathrm{PI} 3 \mathrm{~K} / \mathrm{Akt} / \mathrm{mTOR}$ signaling pathway. In numerous cancer types, perturbations of PTEN regulation or PTEN loss-of-function mutations consequently result in upregulation of $\mathrm{PI} 3 \mathrm{~K} / \mathrm{Akt} / \mathrm{mTOR}$ signaling which contributes, to tumorigenesis (17). The tuberous sclerosis complex (TSC) is a key upstream negative regulator of mTORC1 kinase activity, which exists in a heterodimer that comprises TSC1 and TSC2 (also called harmatin and tuburin, respectively). TSC2 functions as a GTPase-activating protein (GAP) which inhibits the activity of RAS homologue enriched in brain (Rheb) as an essential activator of mTORC1 (18). Activation of RAS-MAPK (mitogenactivated protein kinase) and PI3K/Akt signaling induces the inhibitory phosphorylation of TSC2 and results in dissociation of The TSC1/TSC2 complex, which in turn leads to activation of mTORC1 signaling via Rheb. Thus, suppression of TSC allows the GTP-bound Rheb to bind and activate mTORC1 (Figure 1) $(19,20)$. Under normal conditions, activation of mTORC1 leads to promoting cap-dependent translation initiation and protein synthesis mandatory for cell growth and proliferation, mainly via direct phosphorylation of ribosomal protein S6 kinases (S6Ks) and eukaryotic translation initiation factor (eIF4E)-binding proteins (4E-BPs) $(20,21)$. Phosphorylated S6K1 promotes mRNA translation and cell growth by phosphorylation of ribosomal S6 and eIF-4B. The mTOR-dependent phosphorylation of $4 \mathrm{E}-\mathrm{BP} 1$ potentially disrupts its binding to eIF4E, which also stimulates translation $(12,21)$. mTOR signaling also regulates and activates numerous transcription factors, such as c-MYC, hypoxia-inducible factor $1-\alpha$ (HIF1- $\alpha$ ), STAT3, transcription factor EB (TFEB), peroxisome proliferatoractivated receptor- $\gamma$ (PPAR $\gamma$ ), PPAR $\alpha$, and sterol regulatory element-binding proteins (SREBPs) (22). Also, mTORC1 mediated suppression of autophagy, mainly through the phosphorylation of the serine/threonine kinase Unc-51-like kinase 1 (ULK1), essential core factors of the autophagy, along with the phosphorylation of S6Ks (S6K1 and S6K2) and 4E-BP1, are essential for cell growth (23). In addition, phosphorylation of 


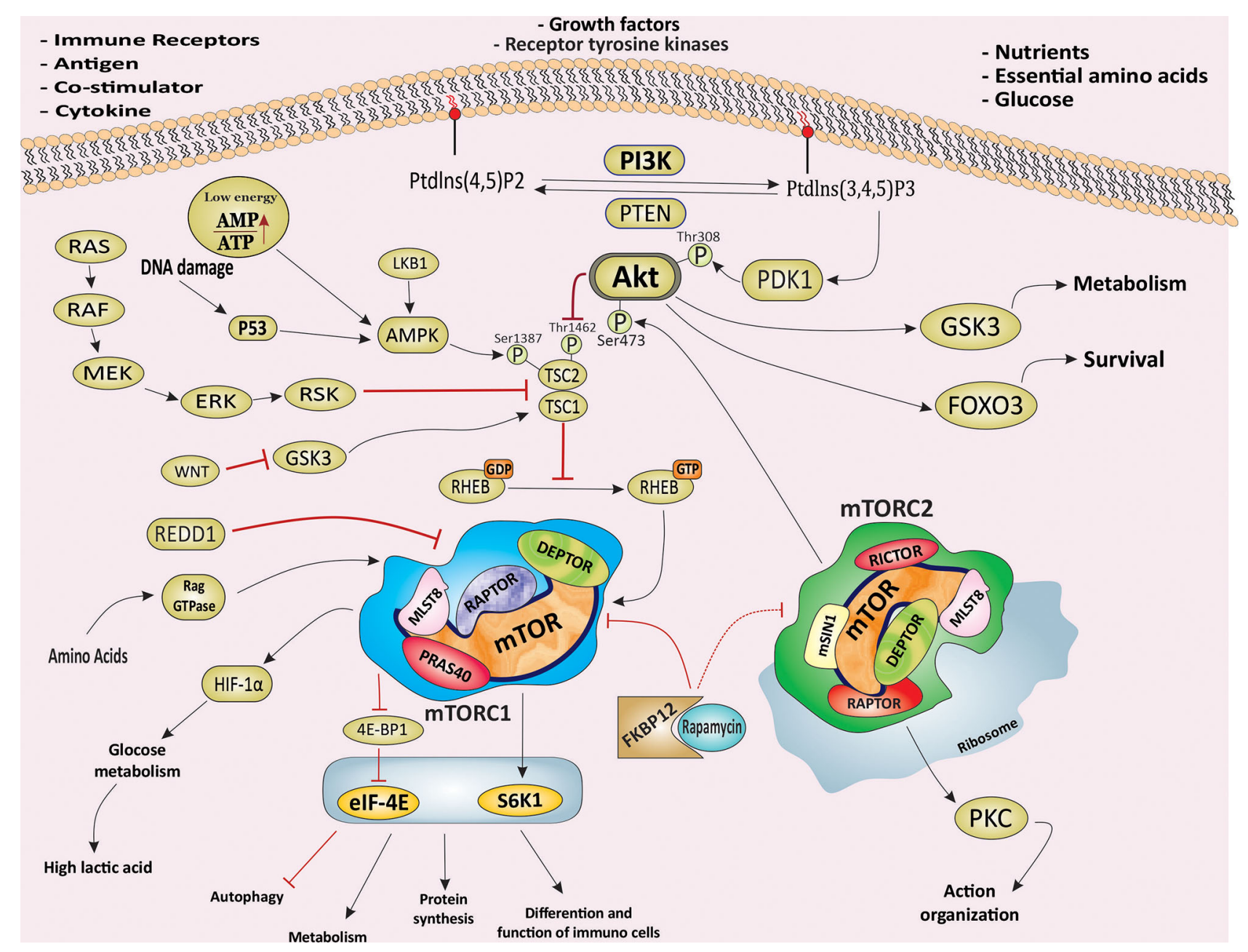

FIGURE 1 | The PI3K/Akt/mTOR signaling pathway and signaling pathway of mTORC1 and mTORC2. Schematic illustration of the molecular components and signaling events related to the PI3K/Akt/mTOR signaling pathway and its main downstream effectors. Schematic showing the main molecular components and signals sensed by mTORC1 and $\mathrm{mTORC} 2$ and the processes they regulate to control main cellular events, including growth, protein synthesis, metabolism, survival and proliferation. mTOR, mechanistic target of rapamycin; PI3K, phosphoinositide 3 kinase; mTORC1, mTOR complex 1; mTORC2, mTOR complex 2; RAPTOR, regulatory-associated protein of mTOR; RICTOR, rapamycin-insensitive companion of mTOR; mSIN1, mammalian stress-activated protein kinase interacting protein; elF4E, eukaryotic initiation factor 4E; 4EBP1, elF4E -binding protein 1; S6K1, S6 kinase 1; HIF-1 $\alpha$, Hypoxia Inducible Factor 1 $\alpha$; PKC, Protein kinase C; AMPK, AMP activated protein kinase; SGK1, glucocorticoid regulated kinase 1; FKBP12, FK506 Binding Protein 12; LKB1, liver kinase B1; DEPTOR, DEP-containing mTOR interacting protein; $\mathrm{mLST} 8$, mammalian lethal with Sec13 protein; RAPTOR, regulatory-associated protein of mTOR; PRAS40, proline-rich AKT substrate $40 \mathrm{kDa}$; mSin1, mammalian stress-activated protein kinase (SAPK)-interacting protein 1; Rheb, RAs homologue enriched in brain; TSC, tuberous sclerosis complex; GSK3, Glycogen synthase kinase-3; PDK1, Phosphoinositide-dependent Kinase 1; FOXO3, Forkhead box family transcription factors 3; PTEN, phosphatase and tensin homologue; REDD1, regulated in development and DNA damage responses 1.

PRAS40 Thr246 by PKB/Akt, and PRAS40 ${ }^{\text {Ser183 }}$ and PRAS40 4 Ser221 by mTORC1, an inhibitory component of mTORC1, leads to the dissociation of the PRAS40 from mTORC1, and its binding to 14-3-3 proteins results in indirect activation of mTORC1, independent of TSC1/2 (24).

Rapamycin (Sirolimus) is a macrolide compound produced by a strain of Streptomyces hygroscopicus, and first discovered in soil samples collected from Rapa Nui (Easter Island) with potent and selective antifungal activity (25). Subsequently, rapamycin and its analogues, Everolimus (RAD001), Temsirolimus (TRM-986), Ridaforolimus (AP23573, MK-8669), and Zotarolimus (ABT-578), were found to have both immunosuppressant and anti-tumor potentials, and they emerged as a promising class of novel antitumor agents $(5,26)$. Rapamycin binds to a cytoplasmic receptor FK506 Binding Protein 12 (FKBP12), and this complex then interacts with the FKBP12-rapamycin-binding (FRB) domain of mTOR and rapidly inhibits mTORC1 downstream signaling (Figure 1) (12). Activation of AMP-activated protein kinase (AMPK) by sensing low cellular energy status and nutrient starvation in the form of intracellular AMP levels, ischemia, as well as under hypoxic conditions leads to an increase in the TSC1/ TSC2-mediated inhibition of Rheb-mTORC1 pathway via 
phosphorylation of TSC2 (27). In addition, glycogen synthase kinase 3 (GSK3)-mediated phosphorylation of TSC2 promotes the inhibition of the mTOR pathway. Wnt signaling inhibits GSK3 resulting in activation of the mTOR pathway (Figure 1) (28). In brief, mTORC1 activation induces cap-dependent translation via activation of at least two independent targets, S6K1 and 4EBP1/ eIF4E, resulting in increases in mammalian cell size and proliferation which are two common features of cancer $(29,30)$.

\section{mTORC2 SIGNALING PATHWAY}

While mTORC1 is highly sensitive to rapamycin-FKBP12 complex, mTORC2 is relatively insensitive to long-term rapamycin treatment and is characterized by its sensitivity to prolonged rapamycin treatment. Rapamycin-mediated inhibition of mTORC2 is thought to be through suppression of mTORC2 assembly, likely as a result of abrogating the binding of rapamycin/ FKBP12 to newly synthesized mTOR to RICTOR (31). Whereas the upstream signals derived from a wide range of extracellular and intracellular causes such as amino acids, growth factors, and cellular functions of mTORC1 are well characterized, little information is known regarding the upstream signals and the biological significance of mTORC2. However, several findings provide new insights into the fundamental role of mTORC2 in the regulation of various biological processes, including cell growth, survival, metabolism, cell migration, proliferation, and cytoskeleton organization and identification of its other physiological and cellular functions are also an open line of investigation $(16,32)$ mTORC2 is a critical regulator of Akt, a crucial serine/threonine kinase in cellular processes and frequently deregulated in many types of human cancer (Figure 1) $(32,33)$. A positive feedback loop between Akt and mTORC2 is necessary for the full activation of Akt. Phosphoinositide-dependent Kinase 1 (PDK1)-dependent phosphorylation of Akt at tyrosine 308 promotes the Akt kinase activity. Subsequently, activated Akt augments mTORC2 kinase activity, resulting in phosphorylation of Akt at serine 473 by mTORC2, which is necessary for full activation of Akt (34). Activated Akt also phosphorylates TSC2, resulting in blockage of TSC2 and TSC1 combination. For downstream effectors, mTORC2 mediated phosphorylation of protein kinase C (PKC) family members, small GTPase RAs homologue (RHO), and serum and glucocorticoid-regulated kinase 1 (SGK1) is a key step in critical cellular processes (Figure 1) (32).

mTORC2 activates $\mathrm{N}$-myc downstream-regulated gene 1 protein (NDRG1) and forkhead box family transcription factors (FOXO), which enhance the cell survival in the response of normal and cellular stresses such as oxidative stress, DNA damage, and nutrient deprivation as well as cancer cells in response to hypoxic stress $(35,36)$. Moreover, mTORC2-mediated activation of PKC family members is involved in regulating cytoskeleton reorganization and cell motility, migration, and invasion involved in tumorigenesis $(37,38)$. It has been demonstrated that PI3K-mediated activation of mTORC2 resulting in enhancing mTORC2- ribosome binding, suggesting that ribosomes activate mTORC2 directly. mTORC2-ribosome interaction subsequently facilitated Akt signaling pathway activation in cancer cells (Figure 1) (39). Further understanding of mTORC2 dysregulation and its physiological functions holds enormous potential to bring regarding that mTORC2 could serve as a novel and amenable therapeutic targets for human disorders, including cancer.

\section{THE CRITICAL ROLE OF MTOR SIGNALING PATHWAY IN CANCER}

Given the crucial role of the mTOR pathway in different fundamental cellular processes, several lines of evidence have identified that the dysregulation of the PI3k/Akt/mTOR signaling pathway closely contributes to the various human pathological conditions including, tumor initiation and progression, maintenance, and metastasis (Table 1) (4-6). Aberrant hyperactivation of the mTOR pathway in cancer mainly results from different levels of mechanisms and its signal cascade (Table 1). First, mutations in the mTOR gene lead to constitutive activation of mTOR, which has been reported in a few human cancers. In a recent study by Grabiner et al., comprehensive cancer-associated mTOR mutations identified thirty-three mutations using publicly available tumor genome sequencing datasets after generating a comprehensive catalogue of mTOR pathway mutations in cancer. The detected mutations clustered in six distinct regions in the C-terminal half of mTOR, and these were accompanied by different cancer types, with one cluster particularly prominent in kidney cancer. Interestingly, these mutations were contributed to the mTOR pathway hyperactivation by inhibiting the interaction of mTOR and its endogenous inhibitor mTOR inhibitor DEPTOR, but did not affect mTOR complex assembly (55). Second, genetic aberrations in the specific components of both mTORC1 and mTORC2 in the non-small cell lung cancer (NSCLC) and breast cancer, respectively, which was associated with poor prognosis and short disease-free survival $(41,56)$. Third, hyperactivation of the mTOR pathway can arise from mutations in upstream elements including, tumor suppressors and oncogenes, which in physiological conditions render it activation or suppression, respectively. Multiple mutations in PI3K signaling pathway members, a bona fide upstream signal pathway of both mTORC1 and mTORC2, have been frequently described in human cancers. Activating mutations and amplification in PIK3CA, the gene encoding the p $110 \alpha$ catalytic subunit of $\mathrm{PI} 3 \mathrm{~K}$ have been reported to frequently mutated in different types of human cancer such as prostate (29\%), breast (27\%), endometrium (23\%), colon (15\%), upper aerodigestive tract, etc (10\%) (46). As a hallmark of proliferating cancer cells, metabolic reprogramming is a critical strategy of cancer cells to alter their metabolism and promote their biological capabilities to ensure their growth, survival, and rapid proliferation. As a master regulator of cellular metabolism, mTOR-mediated upregulation of protein synthesis at the level of S6K1 and 4E-BP1/eIF-4E plays a crucial role in this scenario (57). Glutamine is necessary as a 
TABLE 1 | The role of PI3K/Akt/mTOR signaling pathway components in cancer.

\begin{tabular}{|c|c|c|}
\hline Genes & Consequences of the alteration & Refs \\
\hline Akt & Amplification and overexpression of Akt have been reported in many cancer types. & $(40)$ \\
\hline Rictor & $\begin{array}{l}\text { Rictor is amplified in a subset of human cancers, such as lung and breast cancers, and is associated with cancer progression and therapeutic } \\
\text { resistance. There is an association between RICTOR amplification and sensitivities to mTOR1/2 inhibitors in non-small cell lung cancer cells. }\end{array}$ & $(41-43)$ \\
\hline PIЗK & $\begin{array}{l}\text { PI3K mutations and activity is associated with cell transformation, cancer initiation, and tumor progression. High PI3K activity has been reported } \\
\text { in many human cancers such as ovarian, gastrointestinal, pancreatic, breast, and prostate. }\end{array}$ & $(40,44-46)$ \\
\hline PTEN & $\begin{array}{l}\text { PTEN is a tumor-suppressor gene that acts as a major regulator of the PI3K/Akt/mTOR signaling pathway. Impaired PTEN function results in } \\
\text { tumor initiation and progression and has been described in a large proportion of human cancers. }\end{array}$ & (46) \\
\hline Rheb & $\begin{array}{l}\text { Rheb is the major activator of mTORC1 and frequently overexpressed in human carcinomas and induces multistage carcinogenesis through } \\
\text { induction of multiple oncogenic mechanisms. }\end{array}$ & $(47)$ \\
\hline TSC1/ & Loss of function mutations in TSC1 and TSC2 genes results in constitutive mTOR activation and tumor progression. & $(48)$ \\
\hline \multicolumn{3}{|c|}{ ( } \\
\hline S6K1 & $\begin{array}{l}\text { S6K1 is considered a critical downstream target for mTOR and is abnormally activated in a wide range of human cancers. Overexpression of } \\
\text { S6K1 is associated with poor prognosis in many cancer types such as esophageal squamous cell carcinoma (ESCC), non-small cell lung cancer } \\
\text { (NSCLC), breast cancer, and nasopharyngeal carcinoma (NPC). }\end{array}$ & $(49,50)$ \\
\hline 4EBP1 & $\begin{array}{l}\text { Overexpression of 4EBP1 has shown to be associated with tumorigenesis and poor prognosis in many human cancers, such as breast, head } \\
\text { and neck, colon, and prostate. }\end{array}$ & $(51-54)$ \\
\hline
\end{tabular}

nitrogen and carbon donor for the major biosynthetic pathways such as the synthesis of amino acids, lipids, and nucleotides, which are used by cancer cells to replenish the tricarboxylic acid (TCA) cycle metabolites via a process known as anaplerosis (6). mTORC1 promotes glutamine synthesis via positive regulation of glutamate dehydrogenase (GDH) (58) and by repressing sirtuin 4 (SIRT4), a GDH inhibitor (59). mTOR facilitates cancer cell growth and proliferation by promoting glucose metabolism. Aerobic glycolysis has been regarded as a hallmark of cancer cells, and glutamine provides the main source of carbon and nitrogen for facilitating anabolic processes and supporting cell growth (60). mTOR signaling promotes reprogramming of glucose metabolism by increasing the expression of transporter 1 (Glut1) and glucose uptake (61), as well as by inducing the expression of the transcription factors c-MYC and HIF1- $\alpha$ (62), which play a crucial role in the induction of several other glycolytic enzymes such as phosphoglucoisomerase (PGI), phosphofructokinase (PFK) and enolase (ENO) (Figure 2) (6).

Amplified de novo lipogenesis is considered a hallmark of proliferating cancer cells (63). Both mTORC1 and mTORC2 signaling induce lipogenesis and fatty acid uptake, which is required for cell growth and proliferation, through promoting SREBP1 and PPAR $\gamma$, two critical transcription factors which facilitates the expression of several enzymes involved in lipid and cholesterol homeostasis, including ATP citrate lyase (ACLY), Acetyl-Coenzyme A carboxylase 1 (ACC1), fatty acid synthase (FASN) as well as the fatty acid transporter CD36 (Figure 2) (16, 64, 65).

Although the fundamental role of the mTORC1 signaling pathway has been widely studied in various types of cancer, several recent insights into the function of the mTORC2 pathway uncovered the critical roles for mTORC2 in different cancer types such as prostate, breast, lung cancer, glioblastoma (GBM), pancreatic cancer, and T-cell acute lymphoblastic leukemia (T-ALL) (Table 1)
(41, 43, 66-68). Specific ablation of the mTORC2 component, RICTOR, significantly delays pancreatic tumorigenesis. Interestingly, combined inhibition of mTORC1/2 and PI3K significantly prolonged survival in late-stage tumor in-vivo. Suggesting that targeting mTORC2 as a potential therapeutic strategy for the clinical intervention of pancreatic cancer (68). Overexpression of RICTOR has been detected in $74.0 \%$ of gastric cancers (69) and was associated with tumor progression, lymph node metastasis, and poor prognosis in patients with gastric cancer (69). Targeting of mTORC2 either by kinase inhibitors or RICTOR knockdown induces apoptosis of breast cancer cells and suppresses cell migration and metastasis $(70,71)$. Additionally, RICTOR deficiency results in a substantial decrease in pAktSer ${ }^{473}$ level and significantly reduces the proliferation of colorectal cancer cells and tumor growth (72). Glucose-induced RICTOR acetylation promotes mTORC2 activation, and driving therapeutic resistance to inhibitors of the PI3K pathway result in promoting epidermal growth factor receptor vIII (EGFRvIII)-dependent signaling in glioblastoma cells (73). RICTOR upregulation was found to be contributed to the hyperactivation of Akt, aggressive breast cancers, and decreased overall survival. Additionally, ablation of RICTOR/mTORC2 signaling subsequent RICTOR knockdown or treatment with mTORC1/2 dual kinase reduced Akt-mediated tumor cell survival and promoted lapatinib-mediated cell killing in HER $2^{+}$breast cancer cells, a dual HER2/EGFR tyrosine kinase inhibitor (41). Metabolic reprogramming-mediated by $\mathrm{mTORC} 2$ has been shown to promote tumorigenesis, where it augments tumor growth by providing lipids necessary for growth and energy production. In liver-specific $\mathrm{PTEN}^{-1-}$ and $\mathrm{Tscl}^{-/-}$mouse models, oncogenic activation of mTORC2 resulting in activation of SREBP1 which promotes de novo fatty acid and lipid synthesis such as sphingolipids, glycerophospholipids, and cardiolipins to increase mitochondrial respiration (74). These results highlighs the importance of mTORC2 signaling in cancer, and mTOR inhibition serves as a promising therapeutic strategy for the clinical intervention of cancer. 


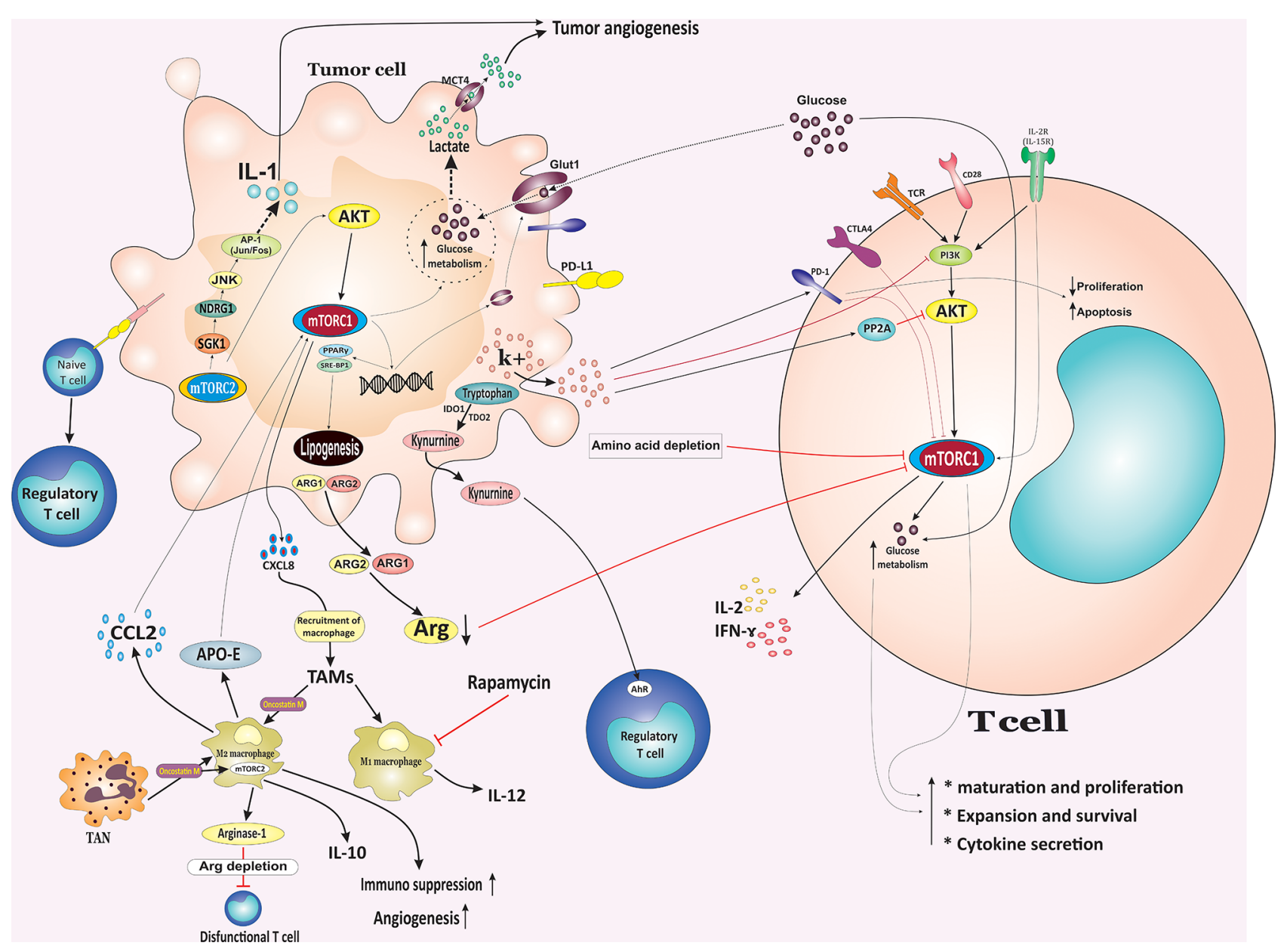

FIGURE 2 | Metabolic competition between tumor cells and T cells in the tumor microenvironment. The nutrient interplay between malignant and nonmalignant cells, especially immune cells, can potentially influence cancer cells growth, survival, and function. Tumors might inhibit immunity through nutrient consumption and simultaneously produce metabolites to inhibit T cell function, resulting in tumor escape from the immune system. In TME, tumor cells outcompete T cells for glucose, leading to sustained mTOR signaling and glycolysis in the tumor cells. Additionally, increased glycolysis by tumor cells leads to the release of lactate via MCT4, which enhances immunosuppression and angiogenesis. In contrast to PD-1 and CTLA-4, which inhibit the mTOR-mediated upregulation of glycolysis, the co-stimulation of the CD28 signaling pathway and signaling mediated via cytokines such as IL-2 and IL-15 activates PI3K/Akt/mTOR pathway to promote the switch of the T cell metabolism to glycolysis. Tumor cells also affect T cells via releasing high-[K+]e, which induced inhibition of TCR-mediated Akt and mTOR phosphorylation and induced the upregulation of PD-1. Cancer cells also evade immune responses by releasing cytosolic ARG1 or mitochondrial ARG2 results in diminished levels of arginine and reduced mTOR activity and inhibition of T cells in the TME. Additionally, upregulation of IDO1 and TDO2 by cancer cells leads to the tryptophan degradation into kynurenine which supports the Treg phenotype in an AhR repentant manner. Overexpression of mTORC2 target SGK1 mediates tumor growth and invasion in different cancer types. Overexpression of the SGK1 target, NDRG1, is suggested to stimulate IL-1 expression and promote tumor angiogenesis through JNK/AP-1 activation. Both mTORC1 and mTORC2 signaling induce lipogenesis and fatty acid uptake through promoting SREBP1 and PPAR $\gamma$. CCL2 secreted by TAMs render cancer cells resistance through activating the PI3K/Akt/mTOR pathway. TME, tumor microenvironment; mTOR, mammalian target of rapamycin; MCT4, monocarboxylate transporter 4; ARG1, arginase 1; IDO1, indoleamine-2,3-dioxygenase 1; TDO2, tryptophan-2,3-dioxygenase 2; AhR, aryl hydrocarbon receptor; Treg, regulatory T cell; SREBP1, sterol regulatory element-binding protein 1; PPAR $\gamma$, peroxisome proliferator-activated receptor- $\gamma$; CCL2, CC-chemokine ligand 2; TAMs, tumor associated macrophages; PD-1, Programmed cell death protein 1; CTLA4, Cytotoxic T-Lymphocyte Associated Protein 4; NDRG1, N-myc downstream-regulated gene 1 protein; MCT4, monocarboxylate transporter 4.

\section{THE PI3K/AKT/mTOR SIGNALING NETWORK AND TUMOR MICROENVIRONMENT}

The TME is complex and composed of diverse cell types and noncellular components such as numerous growth factors, cytokines, chemokines, and other tumor-promoting molecules released or created by the extracellular matrix (75). Nonmalignant cell components of TME include CAFs, adipocytes, vascular endothelial cells, and different immune cell types such as regulatory $\mathrm{T}$ cells (Treg), tumor-associated macrophages (TAM), myeloid-derived suppressor cells (MDSCs), B lymphocytes, dendritic cells (DCs), natural killer (NK) cells and natural killer $\mathrm{T}$ (NKT) cells, $(1,76)$. The nonmalignant cells component of TME 
exhibit a dynamic and often tumor-promoting role at all stages of carcinogenesis (76). Emerging evidence indicates that the crucial regulatory role of the $\mathrm{PI} 3 \mathrm{~K} / \mathrm{Akt} / \mathrm{mTOR}$ axis in differentiation, activation, homeostasis, and effector functional properties of immune cells to coordinate and shape the innate and adaptive immunity $(12,77)$. PI3K/Akt/mTOR axis can sense and integrate inputs from a variety of environmental signals in the context of TME to regulate the immune cell trafficking, polarization, and their functional properties to promote tumor progression and metastasis. Likewise, activation of the PI3K/Akt/mTORC1 pathway is critical for the development and metabolic reprogramming of effector $\mathrm{CD}^{+}$and $\mathrm{CD}^{+} \mathrm{T}$ cells $(78,79)$. Both mTORC1 and mTORC2 mainly function as crucial signaling nodes that receive and integrate multiple upstream input signals from $\mathrm{T}$ cell receptor (TCR) (known as signal 1), several costimulatory molecules (known as signal 2), and different cytokine exposure (known as signal 3) to coordinate the downstream signaling programs for regulating immune receptor signaling pathways, transcriptional and metabolic programming and migratory activity. The signal transduction pathway mediated by mTOR ultimately determines the $\mathrm{T}$ cell homeostatic and dictates immune cell fate decisions in effector, memory, and Tregs cells $(13,80)$. Rheb-deficient $\mathrm{T}$ cells were unable to generate Th1 and Th17 responses in vitro and in vivo and failed to induce classical experimental autoimmune encephalomyelitis (EAE). However, they sustained their ability to become Th2 cells. Alternatively, specific elimination of mTORC2 signaling by selective conditional deletion of the RICTOR in T cells blocked selective Th2 cells development in vitro and in vivo but preserved their ability to differentiate into Th1 and Th17 cells. Additionally, selective deletion of both mTORC1 and mTORC2 signaling were necessary for the generation of immunosuppressive Treg cells in the absence of exogenous transforming growth factor (TGF)$\beta$ (78).

Metabolic reprogramming-mediated by mTOR complexes implicates in orchestrating the interaction of TME components, particularly immune cells and neoplastic cells, suggesting the crucial role of $\mathrm{PI} 3 \mathrm{~K} / \mathrm{Akt} / \mathrm{mTOR}$ in the determination of tumor development, progression as well as drug resistance. Thus, with regard to the many actions in which mTOR is involved, the inhibition of PI3K/Akt/mTOR holds enormous potential to bring about novel therapeutic targets or strategies for reducing cancer cells proliferation, migration, invasion, and survival and enhancing the efficacy of the tumor immunosurveillance through both the downregulation of the immunosuppressive pathways and the activation of anti-tumor immunity in combination with agents able to negate immune suppression and boost $\mathrm{T}$ cell immunity. However, recent studies have demonstrated that the mTOR blockade has surprising immunostimulatory effects by enhancing the generation of memory precursor effector cells that survive and differentiate into long-lived $\mathrm{CD} 8^{+}$memory cells, allowing better clearance of tumor cells (81). Consequently, the pharmacologic or genetic targeting of key components of this signaling pathway is a potential therapeutic target for clinical intervention patients with a range of different cancers.

\section{$\mathrm{CD4}^{+}$SUBSETS AND CD8 ${ }^{+}$ T LYMPHOCYTES AND TUMOR CELLS IN TME: IN COMPETITION FOR NUTRITION}

As was mentioned above, malignant cells undergo metabolic reprogramming characterized by enhancing glucose uptake and aerobic glycolysis, glutamine uptake and glutaminolysis, oxidative phosphorylation (OXPHOS), and lipid metabolism. These metabolic alterations support critical metabolites and energy for rapid malignant proliferation, growth, invasion, metastasis in a nutrient fluctuating environment (6). Similar to cancer cells, immune cells also undergo metabolic reprogramming during development, activation, and effector or memory differentiation, resulting in distinct functional fates (82). $\mathrm{T}$ cells are divided into several distinct subtypes and can destroy target tumor cells directly or indirectly by synthesizing and releasing various biological molecules. In addition, each subset of $\mathrm{T}$ cells exhibits distinct unique metabolic demands for biological energy and biosynthesis and signaling pathways that contribute to its fate and function (82). Naïve T cells undergo extensive changes in their metabolic properties during proliferation, differentiation, and capacity to differentiate into distinct effector subtypes as well as their effector function. Naïve $\mathrm{T}$ cells mostly rely on lower metabolic demand and have a catabolic metabolism by which they use glucose, fatty acids, and amino acids for ATP generation through the TCA cycle and OXPHOS. Upon antigen stimulation, $\mathrm{T}$ cells significantly alter their metabolism to support these increased synthetic demands, and the cells transition into anabolic metabolism mediated by glycolysis and glutaminolysis to obtain energy for cell growth, proliferation, differentiation, and cytokine secretion (80).

In the context of heterogeneous TME, the nutrient interplay between malignant and nonmalignant cells, especially immune cells, can potentially influence tumor cells growth, survival, and function. Tumors might inhibit immunity through nutrient consumption and simultaneously produce metabolites to inhibit $\mathrm{T}$ cell function, resulting in tumor escape from the immune system. Within the TME, tumor cells outcompete $\mathrm{T}$ cells for glucose, leading to sustained mTOR signaling and glycolysis in the tumor cells and, consequently, tumor progression. Conversely, decreased glucose concentrations following enhanced glucose consumption by tumor cells result in downregulation of mTOR activity in antitumor immune cells, glycolysis, and chemokine secretion (83). In turn, the downregulation of mTOR activity in $\mathrm{T}$ cells impairs their metabolic reprogramming and function and facilitates tumor immune escape. Additionally, increased glycolysis by tumor cells leads to the release of lactate via monocarboxylate transporter 4 (MCT4), which promotes immunosuppression and angiogenesis (Figure 2) (84).

Glucose deprivation or reduction in TME following consumption by cancer cells metabolically restricts aerobic glycolysis in tumor-infiltrating $\mathrm{T}$ cells, resulting in $\mathrm{T}$ cell dysfunction and impairing $\mathrm{T}$ cell-mediated immunosurveillance and enhancing immunosuppressive properties of tumorinfiltrating lymphocytes (TILs) $(83,85)$. In addition, glycolysis is obligatory for $\mathrm{T}$ cell maturation, expansion, and effector 
function during an immune response (86). Nutrient competition distinctly can affect TILs activity, and antigen-specific T cell effector function can be affected by tumor cell numbers and glucose concentrations in vivo. In this regard, TILs showed a reduction in mTOR activity and interferon (IFN)-g production. Therefore, mTOR activity may directly or indirectly reflect the nutritional and functional status of immune cells and cancer cells (Figure 2) (83). Ligations of immune checkpoint receptors CTLA4 and PD- 1 have been revealed to suppress the PI3K/Akt/mTOR signaling pathway resulting in diminished IL-2 production, $\mathrm{Bcl}-\mathrm{xL}$ expression, glucose uptake, and glycolytic rate (87). PD-1 is expressed by activated $\mathrm{T}$ lymphocytes and is a crucial immune checkpoint receptor that mediates immunosuppression upon binding to its ligand PD-1 ligand-1 (PD-L1) expressed by tumor cells. They are considered to be the central mediator of immunosuppression in the tumor immune TME. Loss of PTEN expression and subsequently constitutive PI3K/mTOR activation contributed to the upregulating cell surface PD-L1 expression in triple-negative breast cancer (TNBC). Interestingly, treatment of breast cancer cells with either the Akt inhibitor or the mTOR inhibitor was associated with a markedly decrease in PD-L1 cell surface expression (Figure 2). Furthermore, elevated levels of PDL1 expression following PTEN knockdown results in decreased proliferation and increased apoptosis in activated $\mathrm{T}$ cells and raise the possibility that targeting the PI3K/mTOR pathway as a therapeutic strategy may augment adaptive immune responses against cancer (88). Targeting the PD-L1/PD-1 pathway has consistently shown significant and promising therapeutic efficacy in patients with advanced cancers (89). However, PD-L1 is well known to inhibit T cells via PD-1, it is now elucidated that it serves additional biological advantages for tumors. Chang et al. showed that PD-L1 on the tumor cell surface sustains Akt/mTOR signaling, which in turn promotes glycolysis by enhancing the glycolysis enzymes (83). Therefore, PD-L1 blockade therapy reduced mTOR activity and mTOR-mediated upregulation of glycolysis in tumor cells, which, in turn allowing more available glucose within the extracellular milieu of the tumor and restores TILs glycolytic capacity and, as a result, their effector function (83). Hence, anti-PD1 treatment not only blocks inhibitory receptors but also can suppress tumor growth by directly regulating tumor cell metabolism via modulating mTOR activity and reducing the glucose exhaustion in TME, which promotes $\mathrm{CD}^{+}$cytotoxic T lymphocyte (CTL) functions, suggesting a very effective and promising advancement for therapies targeting both tumor immunity and TME.

In contrast to PD- 1 and CTLA- 4 which, inhibit the mTORmediated upregulation of glycolysis, the co-stimulation of CD28 signaling pathway and signaling mediated via cytokines such as IL-2 and IL-15 activates PI3K/Akt/mTOR pathway to promote the switch the T cell metabolism to glycolysis (Figure 2) $(90,91)$. Therefore, preventing the metabolic activity of tumor cells by modulating the PI3K/mTOR pathway may have the potential enormous to enhance glucose availability to $\mathrm{T}$ cells, thereby enhancing the antitumor activity of $\mathrm{T}$ cells.

Tumor cells can also influence the TME by releasing extracellular signals which, derive suppression of $\mathrm{T}$ cell function in the TME. For example, dying or necrotic tumor cells release high- $\left[\mathrm{K}^{+}\right] \mathrm{e}$, which induces the inhibition of TCRmediated Akt and mTOR phosphorylation and upregulates inhibitory protein PD-1 which, resulting in profound effects on tumor-resident $\mathrm{T}$ cell metabolic pathway and induces $\mathrm{T}$ cell suppression (92). The elevated $\mathrm{K}^{+}$concentration leads to more $\mathrm{K}^{+}$entering into the $\mathrm{T}$ cells through a pump or leak channels resulting in an increase in intracellular $\mathrm{K}^{+}$levels. In this milieu, elevated $\mathrm{K}^{+}$drives hypophosphorylation of the Akt/mTOR pathway and suppression of $\mathrm{T}$ cells in a PP2A-dependent manner (Figure 2) (93). PTEN loss and PI3K/Akt pathway activation in melanoma are associated with resistance to $\mathrm{T}$ cellmediated tumor killing, reduced $\mathrm{T}$ cell infiltration at tumor sites, likely due to increased secretion of immunosuppressive cytokines by cancer cells, and resistance to immune checkpoint inhibitors (94). The emerging evidence highlights that sensing of amino acids by the mTOR pathway in immune cells is crucial for their proliferation, metabolism, and activation (95). T cell activation is associated with the rapid uptake of amino acids such as glutamine and leucine that is essential for appropriate metabolic reprogramming (96). Indeed, an influx of branchedchain amino acids (BCAAs) such as leucine and glutamine regulate a broad range of immune cell functions through modulating mTORC1 activation. Sensing of amino acids such as arginine, glutamine, and leucine regulate the recruitment and localization of mTORC1 from the cytoplasm to the lysosomal surface via the RAS-related GTP-binding proteins (RAGs), where enhances mTORC1 activity by bringing the complex in contact with Rheb, which strongly stimulates the kinase activity of mTORC1 (Figure 1) (97). The Rag complex, specially RagD, is required for $\mathrm{CD}^{+} \mathrm{T}$ cell antitumor immunity, and $\mathrm{RagD}$ deficiency induces a dysfunctional phenotype in $\mathrm{CD} 8^{+}$TILs. Amino acids promote the RagD-mediated translocation of mTORC1 to lysosomes, which render the maximal mTORC1 activity in $\mathrm{CD}^{+} \mathrm{TILs}$. Tumor cells reduce $\mathrm{T}$ cell access to leucine and therefore impair leucine-driven $\mathrm{mTORC} 1$ activation, which eventually impairs the CD8 ${ }^{+}$TIL antitumor immunity (98).

Cancer cells activity impacts immune cells by depleting specific amino acids in the tumor milieu and creating an immunosuppressive TME. For example, the release of cytosolic arginase 1 (ARG1) or mitochondrial arginase 2 (ARG2) results in diminished levels of arginine, reduced mTOR activity, and inhibition of T cells in the TME (99). Upregulation of indoleamine-2,3-dioxygenase 1 (IDO1) and tryptophan-2,3dioxygenase 2 (TDO2) by cancer cells result in the tryptophan degradation into kynurenine. While tryptophan deprivation suppresses proliferation and promotes apoptosis in $\mathrm{T}$ cells in the TME, secreted kynurenine supports Treg cells phenotype in an aryl hydrocarbon receptor (AhR) repentant manner, which further suppresses immune responses (100). $\mathrm{T}$ cell suppression may result from a reduction in mTORC1 following a reduction in intracellular amino acid levels. Additionally, overexpression of mTORC2 target SGK1 is correlates with tumor growth and invasion in various cancers (101). In gastric tumors, overexpression of the SGK1 target, NDRG1, is suggested to stimulate IL-1 expression and induce tumor angiogenesis 
through JNK/AP-1 activation. NDRG1 is considered as a metastasis suppressor in many cancer types (102). Additionally, NDRG1 overexpression is associated with enhanced upregulation of angiogenic CXC chemokines, vascular endothelial growth factor A (VEGF-A), matrix metalloproteinase (MMP)-1, and cell invasion, which supports angiogenesis and malignant progression (Figure 2) (103).

Taken together, these data suggest that enhanced PI3K/Akt/ mTOR signaling in cancer cells may affect $\mathrm{T}$ cells fate, and thereby influencing tumorigenesis and malignant progression.

\section{mTOR REGULATES TREG/TH17 BALANCE IN THE TUMOR MICROENVIRONMENT}

Treg cells are an immunosuppressive subset of $\mathrm{CD} 4^{+} \mathrm{T}$ lymphocytes, characterized by the expression master transcription factor forkhead box protein P3 (Foxp3). Foxp $3^{+}$Tregs play an indispensable role in maintaining self-tolerance and cancer immune evasion through their role as a suppressor of the effector T cells. High numbers of tumor-infiltrating Foxp $3^{+}$Tregs and decreased ratios of tumorinfiltrating $\mathrm{CD}^{+} \mathrm{T}$ cells in the tumor infiltrate are correlated with promoting tumor development and progression in several tumor types $(104,105)$. Under normally activating conditions, the reduction in mTOR signaling following mTOR inhibition is associated with Treg expansion. Both pharmacological and genetic disruption of the mTOR signaling induce expansion of the expansion of Tregs by promoting the expression of Foxp3 (106). Conversely, the PI3K/Akt/mTORC1 signaling pathway promotes Th17 cell differentiation. mTOR-dependent upregulation of S6K2 allows S6K2 to interact with ROR $\gamma$, hence accelerating the nuclear translocation of ROR $\gamma$ and Th17 differentiation. Additionally, the $\mathrm{PI} 3 \mathrm{~K} / \mathrm{Akt} / \mathrm{mTORC} 1 / \mathrm{S} 6 \mathrm{~K} 1$ axis induces the downregulation of growth factor-independent protein 1 (Gfil), a negative regulator of Th17 differentiation in an S6K1/2-dependent manner (107).

It has also been confirmed that the metabolic programmingmediated by mTOR in TME can influence the Treg differentiation, motility, and immunosuppressive functions. The migration of activated Treg cells to inflamed tissue, including TME, is critical for their immune-modulatory function. Migration of Treg cells requires glycolysis mediated by the enzyme glucokinase (GCK) induced by a PI3K-mTORC2 pathway. Activation of mTORC2, but not mTORC1, following CD28 stimulation on the surface of activated Treg, induces glucokinase expression. Subsequently, glucokinase induces the rearrangement of the cytoskeleton by triggering intense actin remodeling and ultimately promotes the migration of Tregs into tumor tissues (Figure 3) (108). The toll-like receptor (TLR)mediated activation of the PI3K/Akt/mTORC1 signaling pathway also implicates metabolic reprogramming in Treg cells in TME that promotes Treg cell proliferation. It has been shown that ligation of TLR1 and TLR2 on activated Treg cells activates the PI3K/Akt/mTORC1 signaling pathway, which in turn promotes the expression of glucose transporter Glut1 on the cell membrane. Glut1 subsequently increases glucose uptake and therefore promotes glucose metabolism via glycolysis, supporting the energy demand for Treg cells proliferation and inflammatory function. However, TLR-mediated activation of the PI3K/Akt/mTORC1 signaling pathway impairs the immunosuppressive capacity of Treg cells. Conversely, the transcription factor Foxp3 opposed PI3K/Akt/mTORC1 signaling to reduce glucose uptake, glycolysis, anabolic metabolism while enhancing oxidative and catabolic metabolism. Hence, local inflammatory stimulus and Foxp3 reprogram Treg cell metabolism by balancing mTORC1 signaling and glucose metabolism to promote the proliferation and suppressive function of Treg cells (109). The above findings were consistent with some previous studies, for example, AMPK activation following metabolic stresses such as increased ATP: $\mathrm{ADP}$ ratios during hypoxia suppresses the expression of Glut1 and glycolysis in Tregs by modulating the mTORC1 signaling pathway and reprogramming Treg cell metabolism to promote mitochondrial oxidative metabolism rather than glycolysis (Figure 3) (110).

Interaction of PD-L1 on tumor cells with PD-1 on tumorspecific $\mathrm{T}$ cells promotes differentiation of naïve $\mathrm{CD} 4^{+} \mathrm{T}$ cells into Tregs, leading to immune suppression that supports tumor growth (111). Selective inhibition of mTORC1 signaling via deletion of RAPTOR reduces the PD-1, CTLA-4, and inducible $\mathrm{T}$ cell co-stimulator (ICOS) expression by effector Treg cells (112). It has also been revealed that PD-L1 expression regulates the development, maintenance, and functional properties of induced Treg (iTreg) through enhancing and sustaining the Foxp3 expression and hence, the suppressive function of iTreg cells (Figure 3) (111). Furthermore, both oncogenic and IFN- $\gamma$ mediated membranous expression of PD-L1 is associated with the Akt/mTOR pathway upregulation on NSCLC cells. Further study showed that the combination of mTOR inhibitor rapamycin and a PD-1 blocking antibody efficiently suppressed lung tumor growth (113).

Downregulation of glutamine and leucine metabolism repress the differentiation of Th1 and Th17 effector T cells while preserving Treg differentiation $(96,114,115)$. The activation of the mTORC1 pathway is also essential for the expression of cMyc, which plays a critical role in cell growth, differentiation, and various metabolic activities $(116,117)$. A decrease in the rates of glutamine metabolism led to the downregulation of the mTOR pathway and subsequently less Myc expression, thus resulting in a defect in the upregulation of the metabolic machinery essential for differentiation. In addition, glutaminase converts glutamine into glutamate to support the TCA cycle in growing cells (96, $114,115)$. Glutamate deprivation or amino acid transporter SLC1A5 (ASCT2) deficiency was found to promote Treg cell generation $(96,118)$. In contrast to glutamine, the increased intercellular concentration of L-Arginine directly enhances remarkable metabolic reprogramming and survival capacity of $\mathrm{CD}^{+}$and $\mathrm{CD}^{+} \mathrm{T}$ cells, independently of mTOR signaling or downstream metabolites, thus, improves anti-tumor activity (119). Interestingly, elevated intracellular L-Arginine levels suppress $\mathrm{T}$ cell differentiation, improve $\mathrm{T}$ cell survival and 


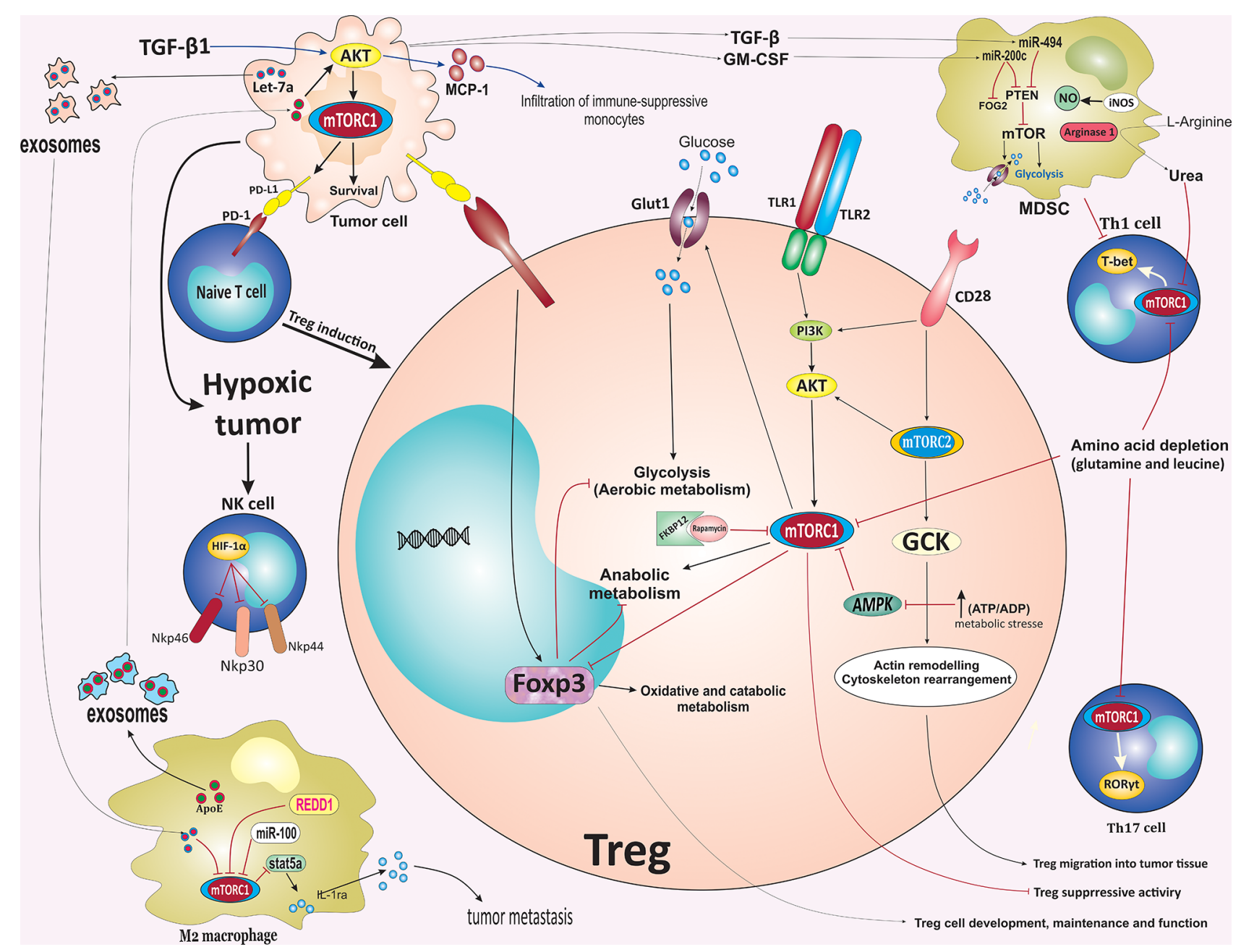

FIGURE 3 | mTOR-mediated regulation of immune cells fate in the tumor microenvironment. Activation of mTORC2 following CD28 stimulation induces GCK expression. GCK promotes the migration of Tregs into tumor tissues via inducing rearrangement of the cytoskeleton. Inflammatory stimulus and Foxp3 reprogramming Treg cell metabolism by balancing mTORC1 signaling and glucose metabolism to promote the proliferation and suppressive function of Treg cells. TLR-mediated activation of mTOR impairs Treg cells suppressive capacity. In contrast to the mTOR pathway, Foxp3 reduces glucose uptake, glycolysis, and anabolic metabolism while enhancing oxidative and catabolic metabolism. AMPK activation following metabolic stresses such as increases in ATP: ADP ratio during hypoxia suppresses the expression of Glut1 and glycolysis in Tregs by modulating the mTORC1 signaling pathway, which reprograms Treg cell metabolism to promote mitochondrial oxidative metabolism rather than glycolysis. Interaction of PD-L1 on tumor cells with PD-1 on tumor-specific T cells promotes differentiation of naive CD4 ${ }^{+} \mathrm{T}$ cells into Tregs. Downregulation of glutamine and leucine metabolism support Tregs differentiation while repressing the differentiation of Th1 and Th17 effector. In the TME, the upregulation of ARG-1 and iNOS expression in MDSCs results in the depletion of L-Arginine. ARG1-mediated hydrolysis of L-Arginine leads to urea production, which subsequently induces metabolic reprogramming of various cellular components in TME, especially T cells, by suppressing the mTOR signaling pathway. TAMs-derived exosomes enriched ApoE promotes the migration of gastric cancer cells by activating the PI3K/Akt/mTOR signaling pathway. Additionally, PI3K/ Akt/mTOR pathway increases the infiltration of immune-suppressive monocytes into tumor sites via inducing the expression of MCP-1 and IL-10, mainly through TGF- $\beta 1$. Hypoxia-induced tumor exosomes that contain let-7a miRNA promote OXPHOS activity and downregulate insulin/Akt/mTOR signaling pathway in bone marrow-derived macrophages and promote polarization of infiltrating macrophages to an M2-like phenotype. mTOR inhibition suppressed the immunosuppressive function of MDSCs via blocking the iNOS pathway and ARG-1 activity. TAM, tumor microenvironment; GCK, glucokinase; mTOR, mammalian target of rapamycin; TLR, Toll-like receptor; Treg, regulatory T cell; Glut1, glucose transporter 1; ARG1, arginase 1; iNOS, inducible nitric oxide synthase; ApoE, apolipoprotein E; MCP-1, monocyte chemoattractant protein-1; ARG1, arginase 1; MDSC, myeloidderived suppressor cells; PD-1, Programmed cell death protein 1; OXPHOS, oxidative phosphorylation; REDD1, regulated in development and DNA damage responses 1; TGF- $\beta 1$, Transforming growth factor $\beta 1$; Foxp3, factor forkhead box protein P3.

sustain cells in a $\mathrm{T}$ central memory-like state. In the TME, the upregulation of ARG-1 and inducible nitric oxide synthase (iNOS) expression in MDSCs results in the depletion of Larginine in the microenvironment which is necessary for $\mathrm{T}$ cell proliferation (120). ARG-1-mediated hydrolysis of L-arginine leads to the production of urea which subsequently induces metabolic reprogramming of various cellular components of local TME, especially T cells, by suppressing the mTOR pathway (Figure 3) (121). Hence, the beneficial influence of LArginine on $\mathrm{T}$ cell survival and anti-tumor functionality may be 
exploited therapeutically to enhance treatment regimens and better outcomes for cancer-bearing patients, for instance, to improve adoptive $\mathrm{T}$ cell therapies.

\section{MTOR PATHWAY MEDIATES POLARIZATION OF TUMOR-ASSOCIATED MACROPHAGE INTO M2 MACROPHAGE}

Macrophages are a highly heterogeneous population of immune cells and are distinctly subdivided into M1 and M2 phenotypes. Of these, M1 macrophages (classically activated macrophages), which develop in the presence of intracellular pathogens and their components, such as lipopolysaccharide (LPS) or Th1 cytokines (such as IFN- $\gamma$ and TNF- $\alpha$ ), are pro-inflammatory with cytotoxic properties and responsible for inflammatory signaling, while M2 macrophages (alternatively activated macrophages) that respond to type II cytokines such as IL-4 and IL-13 are anti-inflammatory macrophages that implicate in the restriction of the inflammatory process and prevent tumor cell attack by immune cells (122). Within the context of the TME, macrophages are the main and critical cell populations implicated in the inflammatory process associated with tumor growth and progression (123). TAMs mainly represent an M2like phenotype, the major population of infiltrating inflammatory immune cell components of the TME, and play a key role in promoting tumorigenesis through mechanisms such as stimulation of angiogenesis, enhancement of tumor migration, immune evasion, chemoresistance, and exert local immunosuppressive effects (124). Metabolic reprogramming-mediated by Akt/mTOR pathway has been shown to be required for determining the activation status of macrophages, the polarization of macrophages toward the M1 or M2 phenotype, and the acquisition of macrophages effector activity, depending on the context in which they are, including the TME (125). Pharmacological inhibition of mTOR by rapamycin has been found to affect macrophage survival and polarization by inducing macrophage apoptosis during M0/M2 and reducing M2 polarization and conversely promoting a shift to an M1-like profile and enhancing M1 phenotype polarization in human and murine macrophages. Beyond the impact on macrophage polarization, mTOR inhibition was associated with modification on macrophage phenotype and cytokine/chemokine secretion profile, with the M2 most profoundly affected $(126,127)$. Accumulation of TAMs associate with tumor progression and angiogenesis. mTOR pathway is crucial element in the regulation of monocyte polarization into TAMs. Rapamycin treatment promotes the differentiation of monocytes into M1 macrophages releasing more IL-12 and less IL-10, whereas TSC2 knockdown-mediated mTOR activation caused the monocytes to differentiate into M2 macrophages releasing less IL-12 and more IL10. Furthermore, infusion of mice with TSC2-deficient or TSC2overexpressing monocytes promote or reduces tumor angiogenesis and growth in murine xenografts by modulating macrophage polarization, respectively (128). Zhao et al. demonstrated that renal cell carcinoma cells are able to recruit macrophages into TME through increasing C-X-C motif chemokine ligand 8 (CXCL8) cytokine expression. Increased infiltrating macrophages were associated with increased renal cell carcinoma cells invasion capabilities and metastasis via inducing the epithelial-mesenchymal transition (EMT) and increased cancer stem cell-like populations by activating the Akt and mTOR signaling pathway (129). In addition, TAMsderived exosomes enriched apolipoprotein $\mathrm{E}$ (ApoE) promote the migration of gastric cancer cells by activation of PI3K/Akt/mTOR signaling pathway (Figure 3) (130).

M1 and M2 macrophages require distinct metabolic programs. While M1 macrophages are known to rely on aerobic glycolysis and lipogenesis programs, M2 macrophages increase glucose utilization, upregulate fatty acid oxidation (FAO), and oxidative phosphorylation (OXPHOS) $(131,132)$. Macrophage colony-stimulating factor (M-CSF) synergizes with IL-4 couple mTORC2 activation with STAT6 signaling which enhances glycolysis during M2 macrophage activation via the induction of the transcription factor interferon regulatory factor 4 (IRF4) (132). Additionally, PI3K/Akt signaling axis was shown to increase the infiltration of immune-suppressive monocytes to tumors via monocyte chemoattractant protein-1 (MCP-1) expression and IL-10, mainly through TGF- $\beta 1$ (Figure 3) (133).

Immunosuppressive TME contains a large number of arginine1 positive $\left(\mathrm{ARG}^{+}\right)$macrophages $(121,134)$. ARG1 production by macrophages stimulated via Th2 cytokines, myeloid suppressor cells, and peripheral myeloid cells in the TME downregulates CD3 $\zeta$ expression, a hallmark of $\mathrm{T}$ cell dysfunction in cancer patients, which results in suppression of TCR expression and antigenspecific $\mathrm{T}$ cell response (135-137). Additionally, argininestarvation arrests $\mathrm{T}$ cells in the G0/G1 phase of the cell cycle as a result of an impaired expression of cyclin D3 and cyclin-dependent kinase 4 (cdk4) in T cells by reducing mRNA stability and ultimately downregulation of translational rate $(138,139)$. These results may help develop novel immunotherapeutic avenues to target arginase as an important step in the success of immunotherapy. TAMsderived products can mediate the activation of PI3K/Akt/mTOR, which, in turn, is associated with drug resistance cancer.For example, enhanced CC-chemokine ligand 2 (CCL2) secretionby TAMs increases endocrine resistance in breast cancer cells via activation of the PI3K/Akt/mTOR signaling pathway. Reciprocally, endocrine-resistant breast cancer cells activate the mTORC1-FOXK1 pathway of macrophages by altering amino acid metabolism in the microenvironment, which enhances M2 macrophage polarization and CCL2 secretion by macrophages. Thus, CCL2 plays a critical role in this malignant feedback loop. Additionally, a high expression level of CCL2 in the stroma is associated with infiltration of $\mathrm{CD} 63^{+}$macrophages and poor progression-free survival (PFS) of patients with estrogen receptorpositive breast cancer. CD163 is a member of the scavenger receptor cysteine-rich (SRCR) protein family, which is considered a highly specific monocyte/macrophage marker for polarization of M2-type macrophages (140). Interestingly, IL-4 signaling co-opts the AktmTOR signaling axis to induce the expression of a subset of M2associated genes by promoting the glucose uptake, glycolysis, and production of cytosolic acetyl-CoA. An increased acetyl-CoA activity mediates epigenetic reprogramming through gene- 
specific histone acetylation to control M2 polarization and activation (141).

Hypoxia conditions have been revealed to promote tumor secretion of exosomes enriched in immunosuppressive components. Hypoxiainduced tumor exosomes containing let-7a microRNA (miRNA) enhance mitochondrial OXPHOS activity and suppress insulin/Akt/ mTOR signaling pathway in bone marrow-derived macrophages (BMMs), as a result, promotes polarization of infiltrating macrophages to an M2-like phenotype. Therefore, biomoleculeloaded exosomes, such as let-7a, enhance tumor immune evasion and tumor progression by promoting changes in immunometabolic profile of infiltrating monocyte-macrophage population (Figure 3) $(2,142)$. miR-100 is highly expressed in TAMs and promotes M2polarization of macrophages, and maintains TAMs phenotype through the downregulation of the mTOR signaling pathway. Furthermore, elevated expression of miR-100 in TAMs is associated with secretion of immunosuppressive cytokine IL-1ra through stat5amediated transcriptional regulation, which promotes cell stemness and tumor metastasis via stimulating the Hedgehog signaling pathway. These findings highlight that the mTOR pathway/miR-100/IL-1ra axis may play an essential role in maintaining the TAMs phenotype and promoting tumor metastasis (Figure 3) (143).

miR-484 exhibits significant anticancer properties by indirectly affecting mTOR-mediated macrophage activation via inhibition of CD137L. CD137L promotes cell viability via the PI3K and mTOR cell pathways and increases IL-8 (CXCL8) production, which results in promoted recruitment of macrophages and neutrophils into the TME (144).

Hypoxic TAMs acquire metabolic changes that promote their angiogenic and immunosuppressive properties. REDD1, a negative regulator of mTOR significantly upregulates in hypoxic TAMs results in suppressing glycolysis in TAMs and inhibiting their excessive angiogenic response. REDD1 deletion in TAMs increases glucose uptake by upregulating Glut1 and glycolysis in an mTOR-dependent manner. These events enhance their rates of glycolysis to a level that competes with neighbouring endothelial cells for glucose, resulting in reducing endothelial glucose availability and persuading them for competition with TAMs for glucose uptake. This metabolic competition over glucose promotes the formation of organized tumor vasculature restores oxygenation within the tumor, and prevents metastases. These results exhibit the functional link between TAM metabolism in hypoxia and tumor angiogenesis (145).

In this regard, targeting the mTOR pathway can be used as a potential therapeutic strategy in an attempt to modulate macrophage responses in the context of TME to promote antitumor immunity with therapeutic benefit in a broad range of cancers.

\section{MTOR PATHWAY DRIVES TUMOR- INDUCED MYELOID-DERIVED SUPPRESSOR CELLS ACCUMULATION TO INDUCE TUMOR PROGRESSION}

MDSCs represent a population of a heterogeneous group of immature myeloid cells defined as $\mathrm{CD}_{11 b^{+}} \mathrm{Grl}^{+}$cells that are characterized by the ability to suppress both adaptive and innate immunities during cancer, infection, and inflammatory diseases. Based on Ly6G and Ly6C expression, MDSCs can be further stratified into two distinct subsets of $\mathrm{CD}_{11 \mathrm{~b}^{+}} \mathrm{Ly}_{6 \mathrm{G}^{+}} \mathrm{Ly}_{6 \mathrm{C}}{ }^{\text {low }}$ granulocytic MDSC (G-MDSC) and $\mathrm{CD}_{11 b^{+}} \mathrm{Ly}_{6 \mathrm{G}}{ }^{+} \mathrm{Ly}_{6} \mathrm{C}^{\text {high }}$ monocytic MDSC (M-MDSC) cells. MDSCs are known as 'queen bee' of TME as they can suppress both innate and adaptive immunity through diverse mechanisms, especially to suppress $\mathrm{T}$ cell responses $(146,147)$. Recently, mTORC1 has been demonstrated to play a pivotal role in the differentiation and function of MDSCs. Specific inhibition of mTORC1 signaling by either immunosuppressant drug rapamycin or genetic deletion decreased the differentiation and accumulation of M-MDSCs in tumors and skin allografts. Alternatively, in conditional disruption of mTORC2 signaling via deletion of RICTOR, the differentiation of MDSCs remained unaffected. Furthermore, mTOR inhibition suppressed the immunosuppressive function of MDSCs via blocking the iNOS pathway and ARG-1 activity. Additionally, mTORC1 activity is necessary for fine-tuning the immunosuppressive $\mathrm{CD} 11 \mathrm{~b}^{+}$ Ly6C ${ }^{\text {high }}$ M-MDSC maturation and their functional properties by enhancing cellular glycolysis activity (Figure 3) (146). Tumorinfiltrating M-MDSCs represent a high level of mTOR phosphorylation. In turn, mTOR-mediated glycolysis is associated with the promoted suppressive function of tumor-infiltrating $\mathrm{M}$ MDSCs (146). In the TME, the upregulation of ARG-1 and iNOS expression in MDSCs leads to the depletion of L-Arginine in the microenvironment, which is necessary for T cell proliferation (120). Further studies for identification of the role of $\mathrm{mTOR}$ in the differentiation of MDSCs showed that pharmacological inhibition of mTOR by rapamycin significantly reduces glucose uptake and lactate production during MDSCs induction. Rapamycin treatment reduces the expression of genes encoding glycolytic enzymes, including the transporter Glut1 and glycolytic enzymes hexokinase 2 (HK2), phosphofructokinase 1 (PFK1), pyruvate kinase muscle

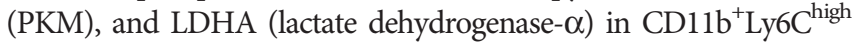
M-MDSCs (Figure 3) (148). mTOR-mediated upregulation of granulocyte colony-stimulating factor (G-CSF) in tumor-initiating cells (TICs) dictates the recruitment of pro-tumorigenic MDSCs into TME. Accumulated MDSCs reciprocally increase TICs frequency by upregulating Notch signaling within tumor cells, suggesting a potential role of mTOR-G-CSF axis in creating a feed-forward loop between TICs and within the TIME. Furthermore, rapamycin-mediated inhibition of mTOR leads to reduced TIC levels (149). TGF- $\beta$ is another factor that promotes the recruitment of MDSCs to the TME. TGF- $\beta$ directly induces differentiation of MDSCs into $\mathrm{CD} 39^{\text {high }} / \mathrm{CD} 73^{\text {high }}$ pro-tumorigenic terminally differentiated myeloid mononuclear cells (TDMMCs) (150). $\mathrm{CD} 9^{+} \mathrm{CD}^{+} 3^{+}$MDSCs have been reported to be dominantly accumulated in the tumor and peri-tumoral stroma of NSCLC patients which is characterized by enriched suppressive molecular signatures. TGF- $\beta$-mediated upregulation of mTOR resultes in the activation of hypoxia-inducible factor- $1 \alpha$ (HIF- $1 \alpha)$ that induces CD39/CD73 expression on tumor-infiltrating MDSCs. Indeed, rapamycin-mediated inhibition of mTOR abrogates the TGF- $\beta$ mediated induction of CD39/CD73 expression on MDSCs by disruption of HIF-1 $\alpha$ (151). Additionally, tumor-infiltrated 
MDSCs can dictate their tumor-promoting effect by determining the fate of the TME components. It has been shown that conditioning of T cells with MDSCs leads to reduced mTOR activity in T cells as well as increased adoptive $\mathrm{T}$ cell-based immunotherapy (ACT) antitumor efficacy, suggesting the critical role of MDSCs in mTORmediated $\mathrm{CD}^{+} \mathrm{T}$ cells differentiation into effector populations (152). However, several lines of evidence have identified that the mTOR inhibition promotes the development of memory $\mathrm{CD}^{+} \mathrm{T}$ cells $(13$, 77, 81).

Tumor-derived factors also play an indispensable role in the upregulation of specific miRNAs in MDSCs to regulate molecular networks controlling the accumulation and function of tumorinfiltrating MDSCs. TGF- $\beta 1$-mediated upregulation of miR-494 in MDSCs supports tumor cells proliferation and metastasis by regulating the activity of MDSCs via targeting mTOR inhibitor PTEN. Similarly, tumor-derived granulocyte-macrophage colonystimulating factor (GM-CSF) induces the expression of miR-200c in tumor environment, and miR-200c, in turn, enhances the expansion and immune suppressive potential activity of MDSCs through negatively targeting PTEN and friend of Gata 2 (FOG2) expression. FOG2 and PTEN downregulation lead to activation of the PI3K/Akt/mTOR signaling pathway to enhance the expansion and immune suppressive potential of MDSCs (Figure 3) (153). Thus, tumor-derived factors induced by miRNAs support the tumor growth and progression by regulating the activity of MDSCs via targeting PTEN, which activates the Akt/mTOR signaling axis.

Taken together, these findings highlight the potential role of mTOR signaling in the differentiation and recruiting protumorigenic MDSCs cells to dictate an immunosuppressive tumor environment.

\section{mTOR-DEPENDENT MECHANISMS IN TUMOR MICROENVIRONMENTS POLARIZE NEUTROPHILS TOWARD PRO-TUMORAL PHENOTYPES}

Neutrophil granulocytes have long been regarded as the most abundant type of granulocytes and the first line of defence against infections and inflammatory conditions. Whereas, several reports have provided evidence for tumor-associated neutrophils (TANs) with potential anti-tumor and anti-metastatic properties (N1 phenotype) with direct cytotoxicity toward tumor cells, others have reported their pro-tumor functions (N2 phenotype) such as supporting angiogenesis, degrading extracellular matrix, promoting the migratory and invasive potential of tumor cells, and shaping the TME toward a more immunosuppressive state (154). Both mTORC1 and mTORC2 complexes are remarkably implicated in the regulation of numerous neutrophil functions, such as chemotaxis, neutrophil extracellular trap (NET) formation, and expression of pro-inflammatory cytokines (12). mTORC2-dependent regulation of Myosin II is essential for neutrophil polarity and migration (155). It has been exhibited that TME resident immune cell subpopulations such as TAMs and TANs secret soluble factors such as Oncostatin M (OSM), an inflammatory cytokine belonging to the IL-6 superfamily, which in turn, promotes polarization of TAMs into pro-tumorigenic M2 phenotype in a mTORC2/Akt1-dependent manner (Figure 2) (156). However, hepatoma-derived soluble factors, including hyaluronan fragments, upregulate the functional LC3 and autophagosomes in neutrophils, resulting in increased autophagy which was unrelated to the deactivation of the mTOR signaling pathway. Interestingly, upregulated neutrophil autophagy was associated with prologue production of pro-metastatic OSM and MMP-9 and promoted metastasis (157). Senescence-associated secretory phenotype (SASP) process refers to the accumulation of proinflammatory mediators and growth-promoting factors secreted by senescent cells in the NF-kB and STAT3 signalingdependent manner. Loss-of-function mutations of oncogenic molecules such as P53, RAS, Notch or mTOR pathway cause to an alternative SASP that correlated with the induction of chronic inflammatory conditions providing an immunosuppressive TME. In this context, immunosuppressive myeloid cells, including macrophages and neutrophils, suppress $\mathrm{NK}$ and $\mathrm{CD}^{+} \mathrm{T}$ cellmediated anti-tumor response and hence augment tumorigenesis (158). The mTOR-S6K1 pathway plays a key role in the neutrophil chemotaxis. In the presence of either GM-CSF or IL-8, preincubated neutrophils with rapamycin significantly inhibits neutrophil chemotaxis and chemokinesis. Rapamycin also inhibits GM-CSF-induced enzymatic activity and actin polymerization, a hallmark of leukocyte migration (159).

These findings highlight the critical role of the mTOR pathway in migratory potential and polarization of TANs toward pro-tumoral phenotypes.

\section{mTOR-MEDIATED REGULATION OF DENDRITIC CELLS IN TUMOR MICROENVIRONMENT}

DCs have a wide range of antigen presentations and are essential for the activation of both helper $\mathrm{CD}^{+}$and cytotoxic $\mathrm{CD} 8^{+} \mathrm{T}$ cells, especially for processing tumor antigens and priming antitumor immunity. The PI3K/Akt/mTOR constitutes a critical pathway downstream of the cytokine FMS-like tyrosine kinase 3 ligand (Flt3L) in is important for DCs subsets development and function, particularly for plasmacytoid DCs (pDCs) and $\mathrm{CD}^{+}$ DCs (13). A more recent study showed that treatment of mature bone marrow monocyte (BMM)-derived DCs with various inhibitors of mTOR (mTORi) promote their antigenpresenting and processing abilities, as well as these cells tended to be non-apoptotic by reducing the expression of apoptotic molecules. In addition, the cytotoxic $\mathrm{CD}^{+} \mathrm{T}$ lymphocytesmediated killing of tumor cells increases following activation of mTORi-treated BMM-derived DCs. Interestingly, in vivo study demonstrated that the mice-bearing tumor treated with both mTORi and connective tissue growth factor (CTGF)/E7 DNA vaccine had higher percentages of mature DCs in the TME with better disease control and prolonged survival. These results revealed that utilization of mTOR inhibitor could be a potential pharmacological approach for temporally extending life span, antigen-presenting and antigen processing of DCs to 
improve the therapeutic outcome of cancer immunotherapy (160). Inter-tumoral delivery of mTORC2-deficient DCs (Rictor-/- DCs) showed pro-inflammatory properties and was associated with reduced melanoma tumor growth, increased numbers of INF- $\gamma+$ and granzyme $\mathrm{B}+(\mathrm{GrB}+) \mathrm{CD}^{+}$TILs, and reduced frequency of immunosuppressive MDSCs within TME (161). These findings raise the possibility that therapeutic inhibition of mTORC2 may present an effective strategy to enhance the therapeutic efficacy of DC-based vaccines for cancer immunotherapy. PI3K/Akt/mTOR signaling axis is critically implicated in regulating the metabolic demands essential for DC activation. It has been shown that TLR-driven mTOR signaling negatively regulates costimulatory molecule expression following LPS stimulation of DCs. Indeed, inhibition of mTOR activity through rapamycin augments the expression of costimulatory molecules, extends the life span, and prolonged activation kinetics of activated DC, via induction of glycolytic metabolism. Furthermore, rapamycin treatment improves the capacity of DCs to augment the induction of primary antigen-specific $\mathrm{CD}^{+} \mathrm{T}$ cell responses and induces effective anti-tumor responses by inducing efficient and sustained CTL responses in a therapeutic vaccination treatment model (162). Suggesting that mTOR signaling in DCs is involved in the establishment of an immunosuppressive environment.

\section{mTOR PATHWAY REGULATES NK AND NKT CELLS METABOLIC ACTIVITY AND PROLIFERATION}

NK cells are known to play an essential role in cancer control. IL15 stimulation promotes NK cell metabolism via prompting intracellular mTOR signaling which, is vital for sustaining the NK cells proliferation, metabolism, and for achieving antitumor cell lysis (163). Conversely, TGF- $\beta$-mediated inhibition of mTOR signaling can directly suppress the metabolism and activation of NK cells (164), suggesting an mTOR-dependent immune suppressive role for TGF- $\beta$ in TME. In NK cells, the mTOR pathway is essential for metabolic response by upregulating glucose uptake and glycolysis (163). HIF-1 $\alpha$ expression depends on the mTOR signaling pathway. Hypoxic environments promote the overexpression of HIF-1 $\alpha$ which causes the downregulation of NK activating receptors NKp46, NKp30, NKp44, and NKG2D (165). Additionally, low-arginine or glutamine conditions significantly suppress mTOR signaling within NK cells, which affect c-Myc expression in IL-2/IL-12stimulated NK cells (Figure 3) (166). Interestingly, gastric cancer mesenchymal stem cells (GCMSCs) impair NK cell function through mTOR signaling (167). In addition to the key roles of mTORC1 for NK cell responses, it is worth noting that continuous exposure of NK cells with IL-15, which is essential for stimulating mTORC1 signaling, drives NK cell exhaustion and reduced cytotoxicity (168).

NKT cells are subsets of T lymphocytes and act as a bridge between innate and adaptive immunity. In addition to killing the CD1d expressing tumor cells directly, activated NKT cells are able to promote the killing effects of NK cells and CTLs against tumor cells (169). Lactic acid production and secretion following Warburg glycolysis by cancer cells results in acidification of the TME, suppresses PI3K/Akt/mTOR pathway, and therefore inhibits T cell glycolysis $(170,171)$. However, the influences of TME on NKT cells functions remain to be fully elucidated. It has been shown that the accumulation of lactic acid in TME suppresses IL- 4 and especially diminishes IFN- $\gamma$ productions by NKT cells. NKT cell dysfunctions were restored upon adjusting $\mathrm{PH}$ to neutral values. The further experiment demonstrated that extracellular acidification inhibited NKT cell functions by inhibiting mTOR signaling and nuclear translocation of promyelocytic leukemia zinc finger (PLZF) (172), a critical transcription factor for NKT cells development and effector function (173). mTOR-mediated regulation of PLZF plays a crucial in NKT cell lineage development and effector function (173).

\section{CONCLUSION}

As was mentioned above, it is well established that upregulation of the PI3K/Akt/mTOR network is critical in promoting tumor pathogenesis by shaping the characterization and the activity of the TME's elements specially recruited immune cells. Although inhibition of mTOR promotes cancer cell death by promoting apoptosis and creating nutrient deprivation conditions in cancer cells, mTORC1 blockade can efficiently enhance prolonged protective immunity by stimulating the generation of longlived protective memory $\mathrm{T}$ cells. In addition, utilization of mTOR inhibitors can also affect the fates of other immune cells recruited to TME such as $\mathrm{CD}^{+}$and $\mathrm{CD} 8^{+} \mathrm{T}$ effector cells, Th17, Tregs, and macrophages, all of which appear to utilize this crucial metabolic regulator for their differentiation and function. Improving immune responses by manipulating cellular metabolic pathways in combination with other anti-cancer agents may provide new options for cancer immunotherapy. In general, metabolite levels can be affected in the absence of genetic manipulations, suggesting the promising opportunity and challenge for therapeutic applications. Additionally, our knowledge of the dynamics of the proteome and metabolome during the immune response constitutes a framework for further studies addressing the complex interplay between metabolism and cellular functions. Concerning TME biology and its potential role as a therapeutic target, the balance between different $\mathrm{T}$ cell subsets and recruitment and activation of immunosuppressive myeloid subsets can be altered by multiple tumor-derived factors released in the context of TME, resulting in mTOR-mediated metabolic and transcriptional reprogramming and the establishment of inappropriate immune cell responses. However, further studies are required to examine how extracellular signals affect $\mathrm{mTOR}$ in regulating immune responses in TME. Hence, combining mTOR inhibitors and cell-based immunotherapies in cancer treatment could be therapeutic strategies to increase the antitumor efficacy of immunotherapies. As a consequence, direct manipulation of immune cells metabolism by manipulating the 
$\mathrm{PI} 3 \mathrm{k} / \mathrm{Akt} / \mathrm{mTOR}$ axis has the potential to provide a new avenue for cancer immunotherapy.

\section{AUTHOR CONTRIBUTIONS}

SM, conceptualization, writing-original draft, and figure design. $\mathrm{BM}$, conceptualization, figure design, writing-review and editing. HS, writing-review and editing, and visualization. RA and ST, writing-original draft and editing. WC, project administration,

\section{REFERENCES}

1. Conciatori F, Bazzichetto C, Falcone I, Pilotto S, Bria E, Cognetti F, et al. Role of mTOR Signaling in Tumor Microenvironment: An Overview. Int $J$ Mol Sci (2018) 19:2453-67. doi: 10.3390/ijms19082453

2. Nazari N, Jafari F, Ghalamfarsa G, Hadinia A, Atapour A, Ahmadi M, et al. The Emerging Role of microRNA in Regulating the mTOR Signaling Pathway in Immune and Inflammatory Responses. Immunol Cell Biol (2021) 99:814-32. doi: 10.1111/imcb.12477

3. Huang K, Fingar DC. Growing Knowledge of the mTOR Signaling Network. Semin Cell Dev Biol (2014) 36:79-90. doi: 10.1016/j.semcdb. 2014.09.011

4. Sabatini DM. mTOR and Cancer: Insights Into a Complex Relationship. Nat Rev Cancer (2006) 6:729. doi: 10.1038/nrc1974

5. Dancey J. mTOR Signaling and Drug Development in Cancer. Nat Rev Clin Oncol (2010) 7:209-19. doi: 10.1038/nrclinonc.2010.21

6. Mossmann D, Park S, Hall MN. mTOR Signalling and Cellular Metabolism are Mutual Determinants in Cancer. Nat Rev Cancer (2018) 18:744-57. doi: 10.1038/s41568-018-0074-8

7. Janku F, Yap TA, Meric-Bernstam F. Targeting the PI3K Pathway in Cancer: Are We Making Headway? Nat Rev Clin Oncol (2018) 15:273. doi: 10.1038/ nrclinonc.2018.28

8. Kaymak I, Williams KS, Cantor JR, Jones RG. Immunometabolic Interplay in the Tumor Microenvironment. Cancer Cell (2020) 1:28-37. doi: 10.1016/ j.ccell.2020.09.004

9. Zhang XC, Wang J, Shao GG, Wang Q, Qu X, Wang B, et al. Comprehensive Genomic and Immunological Characterization of Chinese Non-Small Cell Lung Cancer Patients. Nat Commun (2019) 10:1772. doi: 10.1038/s41467019-09762-1

10. Jin J, Zhao Q. Emerging Role of mTOR in Tumor Immune Contexture: Impact on Chemokine-Related Immune Cells Migration. Theranostics (2020) 10:6231-44. doi: 10.7150/thno.45219

11. Taddei ML, Giannoni E, Comito G, Chiarugi P. Microenvironment and Tumor Cell Plasticity: An Easy Way Out. Cancer Lett (2013) 341:80-96. doi: 10.1016/j.canlet.2013.01.042

12. Powell JD, Pollizzi KN, Heikamp EB, Horton MR. Regulation of Immune Responses by mTOR. Annu Rev Immunol (2012) 30:39-68. doi: 10.1146/ annurev-immunol-020711-075024

13. Rostamzadeh D, Yousefi M, Haghshenas MR, Ahmadi M, Dolati S, Babaloo Z. mTOR Signaling Pathway as a Master Regulator of Memory CD8(+) TCells, Th17, and NK Cells Development and Their Functional Properties. J Cell Physiol (2019) 234:12353-68. doi: 10.1002/jcp.28042

14. Kim LC, Cook RS, Chen J. Mtorc1 and Mtorc2 in Cancer and the Tumor Microenvironment. Oncogene (2017) 36:2191-201. doi: 10.1038/onc.2016.363

15. Li N, Chen J, Liu Q, Qu H, Yang X, Gao P, et al. Prognostic Significance and Tumor-Immune Infiltration of mTOR in Clear Cell Renal Cell Carcinoma. PeerJ (2021) 9:e11901. doi: 10.7717/peerj.11901

16. Laplante M, Sabatini DM. mTOR Signaling at a Glance. J Cell Sci (2009) 122:3589-94. doi: 10.1242/jcs.051011

17. Porta C, Paglino C, Mosca A. Targeting PI3K/Akt/mTOR Signaling in Cancer. Front Oncol (2014) 4:64. doi: 10.3389/fonc.2014.00064

18. Manning BD, Cantley LC. Rheb Fills a GAP Between TSC and TOR. Trends Biochem Sci (2003) 28:573-6. doi: 10.1016/j.tibs.2003.09.003 writing-original draft, writing-review and editing. DR, project administration, writing-original draft, figure design, and writingreview and editing. All authors contributed to the article and approved the submitted version.

\section{FUNDING}

This review is supported by a grant from the Medicinal plant research, Yasuj University of Medical Sciences, Yasuj, Iran.

19. Inoki K, Li Y, Xu T, Guan KL. Rheb GTPase Is a Direct Target of TSC2 GAP Activity and Regulates mTOR Signaling. Genes Dev (2003) 17:1829-34. doi: 10.1101/gad.1110003

20. Huang J, Manning BD. The TSC1-TSC2 Complex: A Molecular Switchboard Controlling Cell Growth. Biochem J (2008) 412:179-90. doi: 10.1042/ BJ20080281

21. Wullschleger S, Loewith R, Hall MN. TOR Signaling in Growth and Metabolism. Cell (2006) 124:471-84. doi: 10.1016/j.cell.2006.01.016

22. Laplante M, Sabatini DM. mTOR Signaling in Growth Control and Disease. Cell (2012) 149:274-93. doi: 10.1016/j.cell.2012.03.017

23. Fruman DA, Limon JJ. Akt and mTOR in B Cell Activation and Differentiation. Front Immunol (2012) 3:228. doi: 10.3389/fimmu. 2012.00228

24. Nascimento EB, Snel M, Guigas B, van der Zon GC, Kriek J, Maassen JA, et al. Phosphorylation of PRAS40 on Thr246 by PKB/AKT Facilitates Efficient Phosphorylation of Ser183 by Mtorc1. Cell Signal (2010) 22:9617. doi: 10.1016/j.cellsig.2010.02.002

25. Vezina C, Kudelski A, Sehgal SN. Rapamycin (AY-22,989), a New Antifungal Antibiotic. I. Taxonomy of the Producing Streptomycete and Isolation of the Active Principle. J Antibiot (Tokyo) (1975) 28:721-6. doi: 10.7164/antibiotics.28.721

26. Chen Y, Zhou X. Research Progress of mTOR Inhibitors. Eur J Medicinal Chem (2020) 208:112820. doi: 10.1016/j.ejmech.2020.112820

27. Inoki K, Kim J, Guan KL. AMPK and mTOR in Cellular Energy Homeostasis and Drug Targets. Annu Rev Pharmacol Toxicol (2012) 52:381-400. doi: 10.1146/annurev-pharmtox-010611-134537

28. Inoki $\mathrm{K}$, Ouyang $\mathrm{H}$, Zhu T, Lindvall $\mathrm{C}$, Wang $\mathrm{Y}$, Zhang $\mathrm{X}$, et al. TSC2 Integrates Wnt and Energy Signals via a Coordinated Phosphorylation by AMPK and GSK3 to Regulate Cell Growth. Cell (2006) 126:955-68. doi: 10.1016/j.cell.2006.06.055

29. Fingar DC, Salama S, Tsou C, Harlow E, Blenis J. Mammalian Cell Size Is Controlled by mTOR and its Downstream Targets S6K1 and 4EBP1/Eif4e. Genes Dev (2002) 16:1472-87. doi: 10.1101/gad.995802

30. Dowling RJ, Topisirovic I, Alain T, Bidinosti M, Fonseca BD, Petroulakis E, et al. Mtorc1-Mediated Cell Proliferation, But Not Cell Growth, Controlled by the 4E-BPs. Science (2010) 328:1172-6. doi: 10.1126/science. 1187532

31. Sarbassov DD, Ali SM, Sengupta S, Sheen JH, Hsu PP, Bagley AF, et al. Prolonged Rapamycin Treatment Inhibits Mtorc2 Assembly and Akt/PKB. Mol Cell (2006) 22:159-68. doi: 10.1016/j.molcel.2006.03.029

32. Oh WJ, Jacinto E. mTOR Complex 2 Signaling and Functions. Cell Cycle (2011) 10:2305-16. doi: $10.4161 / \mathrm{cc} \cdot 10.14 .16586$

33. Sparks CA, Guertin DA. Targeting mTOR: Prospects for mTOR Complex 2 Inhibitors in Cancer Therapy. Oncogene (2010) 29:3733-44. doi: 10.1038/ onc.2010.139

34. Yang G, Murashige DS, Humphrey SJ, James DE. A Positive Feedback Loop Between Akt and Mtorc2 via SIN1 Phosphorylation. Cell Rep (2015) 12:93743. doi: 10.1016/j.celrep.2015.07.016

35. Bakker WJ, Harris IS, Mak TW. FOXO3a is Activated in Response to Hypoxic Stress and Inhibits HIF1-Induced Apoptosis via Regulation of CITED2. Mol Cell (2007) 28:941-53. doi: 10.1016/j.molcel.2007.10.035

36. Weiler M, Blaes J, Pusch S, Sahm F, Czabanka M, Luger S, et al. mTOR Target NDRG1 Confers MGMT-Dependent Resistance to Alkylating 
Chemotherapy. Proc Natl Acad Sci USA (2014) 111:409-14. doi: 10.1073/ pnas. 1314469111

37. Gan X, Wang J, Wang C, Sommer E, Kozasa T, Srinivasula S, et al. PRR5L Degradation Promotes Mtorc2-Mediated PKC-Delta Phosphorylation and Cell Migration Downstream of Galpha12. Nat Cell Biol (2012) 14:686-96. doi: $10.1038 / \mathrm{ncb} 2507$

38. Thomanetz V, Angliker N, Cloetta D, Lustenberger RM, Schweighauser M, Oliveri F, et al. Ablation of the Mtorc2 Component Rictor in Brain or Purkinje Cells Affects Size and Neuron Morphology. J Cell Biol (2013) 201:293-308. doi: 10.1083/jcb.201205030

39. Zinzalla V, Stracka D, Oppliger W, Hall MN. Activation of Mtorc2 by Association With the Ribosome. Cell (2011) 144:757-68. doi: 10.1016/ j.cell.2011.02.014

40. Vivanco I, Sawyers CL. The phosphatidylinositol 3-Kinase AKT pathway in human cancer. Nat Rev Cancer (2002) 2(7):489-501. doi: 10.1038/nrc839

41. Joly MM, Hicks DJ, Jones B, Sanchez V, Estrada MV, Young C, et al. Rictor/ mTORC2 Drives Progression and Therapeutic Resistance of HER2Amplified Breast Cancers. Cancer Res (2016) 76:4752-64. doi: 10.1158/ 0008-5472.CAN-15-3393

42. Cheng H, Zou Y, Ross JS, Wang K, Liu X, Halmos B, et al. RICTOR Amplification Defines a Novel Subset of Patients with Lung Cancer Who May Benefit from Treatment with mTORC1/2 Inhibitors. Cancer Discovery (2015) 5(12):1262-70. doi: 10.1158/2159-8290.CD-14-0971

43. Gkountakos A, Pilotto S, Mafficini A, Vicentini C, Simbolo M, Milella M, et al. Unmasking the Impact of Rictor in Cancer: Novel Insights of Mtorc2 Complex. Carcinogenesis (2018) 39:971-80. doi: 10.1093/carcin/bgy086

44. Mukohara T. PI3K Mutations in Breast Cancer: Prognostic and Therapeutic Implications. Breast Cancer (Dove Med Press) (2015) 7:111-23. doi: 10.2147/ BCTT.S60696

45. Payne SN, Maher ME, Tran NH, Van De Hey DR, Foley TM, Yueh AE, et al. PIK3CA Mutations can Initiate Pancreatic Tumorigenesis and are Targetable with PI3K Inhibitors. Oncogenesis (2015) 4:e169. doi: 10.1038/ oncsis. 2015.28

46. Chalhoub N, Baker SJ. PTEN and the PI3-Kinase Pathway in Cancer. Annu Rev Pathol (2009) 4:127-50. doi: 10.1146/annurev.pathol.4.110807.092311

47. Lu ZH, Shvartsman MB, Lee AY, Shao JM, Murray MM, Kladney RD, et al. Mammalian Target of Rapamycin Activator RHEB is Frequently Overexpressed in Human Carcinomas and is Critical and sufficient for Skin Epithelial Carcinogenesis. Cancer Res (2010) 70(8):3287-98. doi: 10.1158/0008-5472.CAN-09-3467

48. Lee CH, Inoki K, Karbowniczek M, Petroulakis E, Sonenberg N, Henske EP, et al. Constitutive mTOR Activation in TSC Mutants Sensitizes Cells to Energy Starvation and Genomic Damage via p53. EMBO J (2007) 26 (23):4812-23. doi: 10.1038/sj.emboj.7601900

49. Zhang S, Hu B, Lv X, Chen S, Liu W, Shao Z. The Prognostic Role of Ribosomal Protein S6 Kinase 1 Pathway in Patients With Solid Tumors: A Meta-Analysis. Front Oncol (2019) 9:390. doi: 10.3389/fonc.2019.00390

50. Van der Hage J, van den Broek L, Legrand C, Clahsen P, Bosch C, RobanusMaandag E, et al. Overexpression of P70 S6 Kinase Protein is Associated with Increased Risk of Locoregional Recurrence in Node-negative Premenopausal early Breast Cancer Patients. Br J Cancer (2004) 90 (8):1543-50. doi: 10.1038/sj.bjc.6601741

51. Musa J, Orth M, Dallmayer M, Baldauf M, Pardo C, Rotblat B, et al. Eukaryotic Initiation Factor 4E-binding Protein 1 (4E-BP1): A Master Regulator of mRNA Translation Involved in Tumorigenesis. Oncogene (2016) 35(36):4675-88. doi: 10.1038/onc.2015.515

52. Karlsson E, Pérez-Tenorio G, Amin R, Bostner J, Skoog L, Fornander T, et al. The mTOR Effectors 4EBP1 and S6K2 are Frequently Coexpressed, and Associated with a Poor Prognosis and Endocrine Resistance in Breast Cancer: A Retrospective Study Including Patients from the Randomised Stockholm Tamoxifen Trials. Breast Cancer Res (2013) 15(5):1-14. doi: $10.1186 / \mathrm{bcr} 3557$

53. Wang Z, Feng X, Molinolo AA, Martin D, Vitale-Cross L, Nohata N, et al. 4E-BP1 Is a Tumor Suppressor Protein Reactivated by mTOR Inhibition in Head and Neck Cancer. Cancer Res (2019) 79(7):1438-50. doi: 10.1158/ 0008-5472.CAN-18-1220

54. Qin X, Jiang B, Zhang Y. 4E-BP1, a multifactor regulated multifunctional protein. Cell Cycle (2016) 15(6):781-6. doi: 10.1080/15384101.2016.1151581
55. Grabiner BC, Nardi V, Birsoy K, Possemato R, Shen K, Sinha S, et al. A Diverse Array of Cancer-Associated MTOR Mutations are Hyperactivating and can Predict Rapamycin Sensitivity. Cancer Discovery (2014) 4:554-63. doi: 10.1158/2159-8290.CD-13-0929

56. Pilotto S, Simbolo M, Sperduti I, Novello S, Vicentini C, Peretti U, et al. OA06. 06 Druggable Alterations Involving Crucial Carcinogenesis Pathways Drive the Prognosis of Squamous Cell Lung Carcinoma (SqCLC). J Thorac Oncol (2017) 12:S266-7. doi: 10.1016/j.jtho.2016.11.260

57. Lien EC, Lyssiotis CA, Cantley LC. Metabolic Reprogramming by the PI3KAkt-mTOR Pathway in Cancer. Metab Cancer (2016) 207:39-72. doi: 10.1007/978-3-319-42118-6_3

58. Csibi A, Lee G, Yoon S-O, Tong H, Ilter D, Elia I, et al. The Mtorc1/S6K1 Pathway Regulates Glutamine Metabolism Through the Eif4b-Dependent Control of C-Myc Translation. Curr Biol (2014) 24:2274-80. doi: 10.1016/ j.cub.2014.08.007

59. Csibi A, Fendt S-M, Li C, Poulogiannis G, Choo AY, Chapski DJ, et al. The Mtorc1 Pathway Stimulates Glutamine Metabolism and Cell Proliferation by Repressing SIRT4. Cell (2013) 153:840-54. doi: 10.1016/j.cell.2013.04.023

60. Vander Heiden MG, Cantley LC, Thompson CB. Understanding the Warburg Effect: The Metabolic Requirements of Cell Proliferation. science (2009) 324:1029-33. doi: 10.1126/science.1160809

61. Buller CL, Loberg RD, Fan M-H, Zhu Q, Park JL, Vesely E, et al. A GSK-3/ TSC2/mTOR Pathway Regulates Glucose Uptake and GLUT1 Glucose Transporter Expression. Am J Physiology-Cell Physiol (2008) 295:C836-43. doi: 10.1152/ajpcell.00554.2007

62. Gordan JD, Thompson CB, Simon MC. HIF and C-Myc: Sibling Rivals for Control of Cancer Cell Metabolism and Proliferation. Cancer Cell (2007) 12:108-13. doi: 10.1016/j.ccr.2007.07.006

63. Menendez JA, Lupu R. Fatty Acid Synthase and the Lipogenic Phenotype in Cancer Pathogenesis. Nat Rev Cancer (2007) 7:763-77. doi: 10.1038/nrc2222

64. Yecies JL, Zhang HH, Menon S, Liu S, Yecies D, Lipovsky AI, et al. Akt Stimulates Hepatic SREBP1c and Lipogenesis Through Parallel Mtorc1Dependent and Independent Pathways. Cell Metab (2011) 14:21-32. doi: 10.1016/j.cmet.2011.06.002

65. Hagiwara A, Cornu M, Cybulski N, Polak P, Betz C, Trapani F, et al. Hepatic Mtorc2 Activates Glycolysis and Lipogenesis Through Akt, Glucokinase, and SREBP1c. Cell Metab (2012) 15:725-38. doi: 10.1016/j.cmet.2012.03.015

66. Masri J, Bernath A, Martin J, Jo OD, Vartanian R, Funk A, et al. Mtorc2 Activity is Elevated in Gliomas and Promotes Growth and Cell Motility via Overexpression of Rictor. Cancer Res (2007) 67:11712-20. doi: 10.1158/ 0008-5472.CAN-07-2223

67. Guertin DA, Stevens DM, Saitoh M, Kinkel S, Crosby K, Sheen JH, et al. mTOR Complex 2 is Required for the Development of Prostate Cancer Induced by Pten Loss in Mice. Cancer Cell (2009) 15:148-59. doi: 10.1016/ j.ccr.2008.12.017

68. Driscoll DR, Karim SA, Sano M, Gay DM, Jacob W, Yu J, et al. Mtorc2 Signaling Drives the Development and Progression of Pancreatic Cancer. Cancer Res (2016) 76:6911-23. doi: 10.1158/0008-5472.CAN-16-0810

69. Bian Y, Wang Z, Xu J, Zhao W, Cao H, Zhang Z. Elevated Rictor Expression is Associated With Tumor Progression and Poor Prognosis in Patients With Gastric Cancer. Biochem Biophys Res Commun (2015) 464:534-40. doi: 10.1016/j.bbrc.2015.07.001

70. Zhang F, Zhang X, Li M, Chen P, Zhang B, Guo H, et al. mTOR Complex Component Rictor Interacts With Pkc $\zeta$ and Regulates Cancer Cell Metastasis. Cancer Res (2010) 70:9360-70. doi: 10.1158/0008-5472.CAN-10-0207

71. Li H, Lin J, Wang X, Yao G, Wang L, Zheng H, et al. Targeting of Mtorc2 Prevents Cell Migration and Promotes Apoptosis in Breast Cancer. Breast Cancer Res Treat (2012) 134:1057-66. doi: 10.1007/s10549-012-2036-2

72. Gulhati P, Cai Q, Li J, Liu J, Rychahou PG, Qiu S, et al. Targeted Inhibition of Mammalian Target of Rapamycin Signaling Inhibits Tumorigenesis of Colorectal Cancer. Clin Cancer Res (2009) 15:7207-16. doi: 10.1158/10780432.CCR-09-1249

73. Masui K, Tanaka K, Ikegami S, Villa GR, Yang H, Yong WH, et al. GlucoseDependent Acetylation of Rictor Promotes Targeted Cancer Therapy Resistance. Proc Natl Acad Sci USA (2015) 112:9406-11. doi: 10.1073/pnas.1511759112

74. Guri Y, Colombi M, Dazert E, Hindupur SK, Roszik J, Moes S, et al. Mtorc2 Promotes Tumorigenesis via Lipid Synthesis. Cancer Cell (2017) 32:807-823. e812. doi: 10.1016/j.ccell.2017.11.011 
75. Li H, Fan X, Houghton J. Tumor Microenvironment: The Role of the Tumor Stroma in Cancer. J Cell Biochem (2007) 101:805-15. doi: 10.1002/jcb.21159

76. Balkwill FR, Capasso M, Hagemann T. The Tumor Microenvironment at a Glance. J Cell Sci (2012) 125(Pt 23):5591-6. doi: 10.1242/jcs.116392

77. Rao RR, Li Q, Odunsi K, Shrikant PA. The mTOR Kinase Determines Effector Versus Memory CD8+ T Cell Fate by Regulating the Expression of Transcription Factors T-Bet and Eomesodermin. Immunity (2010) 32:6778. doi: 10.1016/j.immuni.2009.10.010

78. Delgoffe GM, Pollizzi KN, Waickman AT, Heikamp E, Meyers DJ, Horton MR, et al. The Kinase mTOR Regulates the Differentiation of Helper T Cells Through the Selective Activation of Signaling by Mtorc1 and Mtorc2. Nat Immunol (2011) 12:295. doi: 10.1038/ni.2005

79. Kim EH, Suresh M. Role of PI3K/Akt Signaling in Memory CD8 T Cell Differentiation. Front Immunol (2013) 4:20. doi: 10.3389/fimmu.2013.00020

80. Chi H. Regulation and Function of mTOR Signalling in T Cell Fate Decisions. Nat Rev Immunol (2012) 12:325-38. doi: 10.1038/nri3198

81. Araki K, Turner AP, Shaffer VO, Gangappa S, Keller SA, Bachmann MF, et al. mTOR Regulates Memory CD8 T-Cell Differentiation. Nature (2009) 460:108-12. doi: 10.1038/nature08155

82. Bantug GR, Galluzzi L, Kroemer G, Hess C. The Spectrum of T Cell Metabolism in Health and Disease. Nat Rev Immunol (2018) 18:19. doi: 10.1038/nri.2017.99

83. Chang CH, Qiu J, O'sullivan D, Buck MD, Noguchi T, Curtis JD, et al. Metabolic Competition in the Tumor Microenvironment Is a Driver of Cancer Progression. Cell (2015) 162:1229-41. doi: 10.1016/j.cell.2015.08.016

84. Sun X, Wang M, Wang M, Yao L, Li X, Dong H, et al. Role of ProtonCoupled Monocarboxylate Transporters in Cancer: From Metabolic Crosstalk to Therapeutic Potential. Front Cell Dev Biol (2020) 8:651. doi: 10.3389/fcell.2020.00651

85. Ho PC, Bihuniak JD, Macintyre AN, Staron M, Liu X, Amezquita R, et al. Phosphoenolpyruvate Is a Metabolic Checkpoint of Anti-Tumor T Cell Responses. Cell (2015) 162:1217-28. doi: 10.1016/j.cell.2015.08.012

86. Frauwirth KA, Riley JL, Harris MH, Parry RV, Rathmell JC, Plas DR, et al. The CD28 Signaling Pathway Regulates Glucose Metabolism. Immunity (2002) 16:769-77. doi: 10.1016/S1074-7613(02)00323-0

87. Parry RV, Chemnitz JM, Frauwirth KA, Lanfranco AR, Braunstein I, Kobayashi SV, et al. CTLA-4 and PD-1 Receptors Inhibit T-Cell Activation by Distinct Mechanisms. Mol Cell Biol (2005) 25:9543-53. doi: 10.1128/MCB.25.21.9543-9553.2005

88. Mittendorf EA, Philips AV, Meric-Bernstam F, Qiao N, Wu Y, Harrington S, et al. PD-L1 Expression in Triple-Negative Breast Cancer. Cancer Immunol Res (2014) 2:361-70. doi: 10.1158/2326-6066.CIR-13-0127

89. Jiang X, Wang J, Deng X, Xiong F, Ge J, Xiang B, et al. Role of the Tumor Microenvironment in PD-L1/PD-1-Mediated Tumor Immune Escape. Mol Cancer (2019) 18:10. doi: 10.1186/s12943-018-0928-4

90. Cornish GH, Sinclair LV, Cantrell DA. Differential Regulation of T-Cell Growth by IL-2 and IL-15. Blood (2006) 108:600-8. doi: 10.1182/blood2005-12-4827

91. Ray JP, Staron MM, Shyer JA, Ho PC, Marshall HD, Gray SM, et al. The Interleukin-2-Mtorc1 Kinase Axis Defines the Signaling, Differentiation, and Metabolism of T Helper 1 and Follicular B Helper T Cells. Immunity (2015) 43:690-702. doi: 10.1016/j.immuni.2015.08.017

92. Ong ST, Ng AS, Ng XR, Zhuang Z, Wong BHS, Prasannan P, et al. Extracellular K+ Dampens $\mathrm{T}$ Cell Functions: Implications for Immune Suppression in the Tumor Microenvironment. Bioelectricity (2019) 1:16979. doi: 10.1089/bioe.2019.0016

93. Eil R, Vodnala SK, Clever D, Klebanoff CA, Sukumar M, Pan JH, et al. Ionic Immune Suppression Within the Tumour Microenvironment Limits T Cell Effector Function. Nature (2016) 537:539-43. doi: 10.1038/nature19364

94. Peng W, Chen JQ, Liu C, Malu S, Creasy C, Tetzlaff MT, et al. Loss of PTEN Promotes Resistance to T Cell-Mediated Immunotherapy. Cancer Discovery (2016) 6:202-16. doi: 10.1158/2159-8290.CD-15-0283

95. Ananieva EA, Patel CH, Drake CH, Powell JD, Hutson SM. Cytosolic Branched Chain Aminotransferase (BCATc) Regulates Mtorc1 Signaling and Glycolytic Metabolism in CD4+ T Cells. J Biol Chem (2014) 289:18793804. doi: 10.1074/jbc.M114.554113

96. Nakaya M, Xiao Y, Zhou X, Chang J-H, Chang M, Cheng X, et al. Inflammatory $\mathrm{T}$ Cell Responses Rely on Amino Acid Transporter ASCT2
Facilitation of Glutamine Uptake and Mtorc1 Kinase Activation. Immunity (2014) 40:692-705. doi: 10.1016/j.immuni.2014.04.007

97. Nguyen TP, Frank AR, Jewell JL. Amino Acid and Small GTPase Regulation of Mtorc1. Cell Logist (2017) 7:e1378794. doi: 10.1080/21592799.2017. 1378794

98. Zhang Y, Hu H, Liu W, Yan S-M, Li Y, Tan L, et al. Amino Acids and RagD Potentiate Mtorc1 Activation in CD8+ T Cells to Confer Antitumor Immunity. J Immunother Cancer (2021) 9:e002137. doi: 10.1136/jitc-2020-002137

99. Timosenko E, Hadjinicolaou AV, Cerundolo V. Modulation of CancerSpecific Immune Responses by Amino Acid Degrading Enzymes. Immunotherapy (2017) 9:83-97. doi: 10.2217/imt-2016-0118

100. Pavlova NN, Thompson CB. The Emerging Hallmarks of Cancer Metabolism. Cell Metab (2016) 23:27-47. doi: 10.1016/j.cmet.2015.12.006

101. Sang Y, Kong P, Zhang S, Zhang L, Cao Y, Duan X, et al. SGK1 in Human Cancer: Emerging Roles and Mechanisms. Front Oncol (2021) 10:2987. doi: 10.3389 /fonc. 2020.608722

102. Kovacevic Z, Richardson DRJC. The Metastasis Suppressor, Ndrg-1: A New Ally in the Fight Against Cancer. Carcinogenesis (2006) 27:2355-66. doi: $10.1093 /$ carcin/bgl146

103. Murakami Y, Watari K, Shibata T, Uba M, Ureshino H, Kawahara A, et al. N-Myc Downstream-Regulated Gene 1 Promotes Tumor Inflammatory Angiogenesis Through JNK Activation and Autocrine Loop of Interleukin$1 \alpha$ by Human Gastric Cancer Cells. J Biol Chem (2013) 288:25025-37. doi: 10.1074/jbc.M113.472068

104. Dolati S, Babaloo Z, Ayromlou H, Ahmadi M, Rikhtegar R, Rostamzadeh D, et al. Nanocurcumin Improves Regulatory T-Cell Frequency and Function in Patients With Multiple Sclerosis. J Neuroimmunol (2019) 327:15-21. doi: 10.1016/j.jneuroim.2019.01.007

105. Togashi Y, Shitara K, Nishikawa H. Regulatory T Cells in Cancer Immunosuppression-Implications for Anticancer Therapy. Nat Rev Clin Oncol (2019) 16:356-71. doi: 10.1038/s41571-019-0175-7

106. Thomson AW, Turnquist HR, Raimondi G. Immunoregulatory Functions of mTOR Inhibition. Nat Rev Immunol (2009) 9:324-37. doi: 10.1038/nri2546

107. Kurebayashi Y, Nagai S, Ikejiri A, Ohtani M, Ichiyama K, Baba Y, et al. PI3KAkt-Mtorc1-S6K1/2 Axis Controls Th17 Differentiation by Regulating Gfil Expression and Nuclear Translocation of Ror $\gamma$. Cell Rep (2012) 1:360-73. doi: 10.1016/j.celrep.2012.02.007

108. Kishore M, Cheung KC, Fu H, Bonacina F, Wang G, Coe D, et al. Regulatory $\mathrm{T}$ Cell Migration is Dependent on Glucokinase-Mediated Glycolysis. Immunity (2017) 47:875-889. e810. doi: 10.1016/j.immuni.2017.10.017

109. Gerriets VA, Kishton RJ, Johnson MO, Cohen S, Siska PJ, Nichols AG, et al. Foxp3 and Toll-Like Receptor Signaling Balance T Reg Cell Anabolic Metabolism for Suppression. Nat Immunol (2016) 17:1459-66. doi: 10.1038/ni.3577

110. Hardie DG. AMP-Activated Protein Kinase: An Energy Sensor That Regulates All Aspects of Cell Function. Genes Dev (2011) 25:1895-908. doi: $10.1101 / \operatorname{gad} .17420111$

111. Francisco LM, Salinas VH, Brown KE, Vanguri VK, Freeman GJ, Kuchroo VK, et al. PD-L1 Regulates the Development, Maintenance, and Function of Induced Regulatory T Cells. J Exp Med (2009) 206:3015-29. doi: 10.1084/ jem. 20090847

112. Sun IH, Oh MH, Zhao L, Patel CH, Arwood ML, Xu W, et al. mTOR Complex 1 Signaling Regulates the Generation and Function of Central and Effector Foxp3(+) Regulatory T Cells. J Immunol (2018) 201:481-92. doi: 10.4049/jimmunol.1701477

113. Lastwika KJ, Wilson W3rd, Li QK, Norris J, Xu H, Ghazarian SR, et al. Control of PD-L1 Expression by Oncogenic Activation of the AKT-mTOR Pathway in Non-Small Cell Lung Cancer. Cancer Res (2016) 76:227-38. doi: 10.1158/0008-5472.CAN-14-3362

114. Sinclair LV, Rolf J, Emslie E, Shi Y-B, Taylor PM, Cantrell DA. Control of Amino-Acid Transport by Antigen Receptors Coordinates the Metabolic Reprogramming Essential for T Cell Differentiation. Nat Immunol (2013) 14:500. doi: 10.1038/ni.2556

115. Patel CH, Powell JD. Targeting T Cell Metabolism to Regulate T Cell Activation, Differentiation and Function in Disease. Curr Opin Immunol (2017) 46:82-8. doi: 10.1016/j.coi.2017.04.006

116. Wall M, Poortinga G, Hannan KM, Pearson RB, Hannan RD, Mcarthur GA. Translational Control of C-MYC by Rapamycin Promotes Terminal Myeloid 
Differentiation. J Am Soc Hematol (2008) 112:2305-17. doi: 10.1182/blood2007-09-111856

117. Babcock JT, Nguyen HB, He Y, Hendricks JW, Wek RC, Quilliam LA. Mammalian Target of Rapamycin Complex 1 (Mtorc1) Enhances Bortezomib-Induced Death in Tuberous Sclerosis Complex (TSC)-Null Cells by a C-MYC-Dependent Induction of the Unfolded Protein Response. J Biol Chem (2013) 288:15687-98. doi: 10.1074/jbc.M112.431056

118. Klysz D, Tai X, Robert PA, Craveiro M, Cretenet G, Oburoglu L, et al. Glutamine-Dependent $\alpha$-Ketoglutarate Production Regulates the Balance Between T Helper 1 Cell and Regulatory T Cell Generation. Sci Signal (2015) 8:ra97-7. doi: 10.1126/scisignal.aab2610

119. Geiger R, Rieckmann JC, Wolf T, Basso C, Feng Y, Fuhrer T, et al. L-Arginine Modulates T Cell Metabolism and Enhances Survival and Anti-Tumor Activity. Cell (2016) 167:829-842 e813. doi: 10.1016/j.cell.2016.09.031

120. Gabrilovich DI, Nagaraj S. Myeloid-Derived Suppressor Cells as Regulators of the Immune System. Nat Rev Immunol (2009) 9:162-74. doi: 10.1038/ nri2506

121. Bronte V, Zanovello P. Regulation of Immune Responses by L-Arginine Metabolism. Nat Rev Immunol (2005) 5:641-54. doi: 10.1038/nri1668

122. Saqib U, Sarkar S, Suk K, Mohammad O, Baig MS, Savai R. Phytochemicals as Modulators of M1-M2 Macrophages in Inflammation. Oncotarget (2018) 9:17937-50. doi: 10.18632/oncotarget.24788

123. Balkwill F, Charles KA, Mantovani AJCC. Smoldering and Polarized Inflammation in the Initiation and Promotion of Malignant Disease. Cancer Cell (2005) 7:211-7. doi: 10.1016/j.ccr.2005.02.013

124. Biswas SK, Allavena P, Mantovani A. Tumor-Associated Macrophages: Functional Diversity, Clinical Significance, and Open Questions. Semin Immunopathol (2013) 35:585-600. doi: 10.1007/s00281-013-0367-7

125. Covarrubias AJ, Aksoylar HI, Horng T. Control of Macrophage Metabolism and Activation by mTOR and Akt Signaling. Semin Immunol (2015) 27:28696. doi: 10.1016/j.smim.2015.08.001

126. Yang CS, Song CH, Lee JS, Jung SB, Oh JH, Park J, et al. Intracellular Network of Phosphatidylinositol 3-Kinase, Mammalian Target of the Rapamycin/70 kDa Ribosomal S6 Kinase 1, and Mitogen-Activated Protein Kinases Pathways for Regulating Mycobacteria-Induced IL-23 Expression in Human Macrophages. Cell Micribiol (2006) 8:1158-71. doi: 10.1111/j.1462-5822.2006.00699.x

127. Mercalli A, Calavita I, Dugnani E, Citro A, Cantarelli E, Nano R, et al. Rapamycin Unbalances the Polarization of Human Macrophages to M 1. Immunology (2013) 140:179-90. doi: 10.1111/imm.12126

128. Chen W, Ma T, Shen X-N, Xia X-F, Xu G-D, Bai X-L, et al. MacrophageInduced Tumor Angiogenesis is Regulated by the TSC2-mTOR Pathway. Cancer Res (2012) 72:1363-72. doi: 10.1158/0008-5472.CAN-11-2684

129. Yang Z, Xie H, He D, Li L. Infiltrating Macrophages Increase RCC Epithelial Mesenchymal Transition (EMT) and Stem Cell-Like Populations via AKT and mTOR Signaling. Oncotarget (2016) 7:44478-91. doi: 10.18632/ oncotarget.9873

130. Zheng P, Luo Q, Wang W, Li J, Wang T, Wang P, et al. Tumor-Associated Macrophages-Derived Exosomes Promote the Migration of Gastric Cancer Cells by Transfer of Functional Apolipoprotein E. Cell Death Dis (2018) 9:114. doi: 10.1038/s41419-018-0465-5

131. Jha AK, Huang SC-C, Sergushichev A, Lampropoulou V, Ivanova Y, Loginicheva E, et al. Network Integration of Parallel Metabolic and Transcriptional Data Reveals Metabolic Modules That Regulate Macrophage Polarization. Immunity (2015) 42:419-30. doi: 10.1016/j.immuni.2015.02.005

132. Huang SC-C, Smith AM, Everts B, Colonna M, Pearce EL, Schilling JD, et al. Metabolic Reprogramming Mediated by the Mtorc2-IRF4 Signaling Axis is Essential for Macrophage Alternative Activation. Immunity (2016) 45:81730. doi: $10.1016 /$ j.immuni.2016.09.016

133. Díaz-Valdés N, Basagoiti M, Dotor J, Aranda F, Monreal I, Riezu-Boj JI, et al. Induction of Monocyte Chemoattractant Protein-1 and Interleukin-10 by Tgf $\beta 1$ in Melanoma Enhances Tumor Infiltration and Immunosuppression. Cancer Res (2011) 71:812-21. doi: 10.1158/0008-5472.CAN-10-2698

134. Marigo I, Zilio S, Desantis G, Mlecnik B, Agnellini AHR, Ugel S, et al. T Cell Cancer Therapy Requires CD40-CD40L Activation of Tumor Necrosis Factor and Inducible Nitric-Oxide-Synthase-Producing Dendritic Cells. Cancer Cell (2016) 30:377-90. doi: 10.1016/j.ccell.2016.08.004
135. Alderton WK, Cooper CE, Knowles RG. Nitric Oxide Synthases: Structure, Function and Inhibition. Biochem J (2001) 357:593-615. doi: 10.1042/ bj3570593

136. Rodriguez PC, Quiceno DG, Zabaleta J, Ortiz B, Zea AH, Piazuelo MB, et al. Arginase I Production in the Tumor Microenvironment by Mature Myeloid Cells Inhibits T-Cell Receptor Expression and Antigen-Specific T-Cell Responses. Cancer Res (2004) 64:5839-49. doi: 10.1158/0008-5472.CAN04-0465

137. Zea AH, Rodriguez PC, Atkins MB, Hernandez C, Signoretti S, Zabaleta J, et al. Arginase-Producing Myeloid Suppressor Cells in Renal Cell Carcinoma Patients: A Mechanism of Tumor Evasion. Cancer Res (2005) 65:3044-8. doi: 10.1158/0008-5472.CAN-04-4505

138. Rodriguez PC, Quiceno DG, Ochoa AC. L-Arginine Availability Regulates TLymphocyte Cell-Cycle Progression. Blood (2007) 109:1568-73. doi: 10.1182/blood-2006-06-031856

139. Rodriguez PC, Hernandez CP, Morrow K, Sierra R, Zabaleta J, Wyczechowska DD, et al. L-Arginine Deprivation Regulates Cyclin D3 mRNA Stability in Human T Cells by Controlling HuR Expression. J Immunol (2010) 185:5198-204. doi: 10.4049/jimmunol.1001224

140. Li D, Ji H, Niu X, Yin L, Wang Y, Gu Y, et al. Tumor-Associated Macrophages Secrete CC-Chemokine Ligand 2 and Induce Tamoxifen Resistance by Activating PI3K/Akt/mTOR in Breast Cancer. Cancer $S c i$ (2020) 111:47-58. doi: 10.1111/cas.14230

141. Covarrubias AJ, Aksoylar HI, Yu J, Snyder NW, Worth AJ, Iyer SS, et al. AktMtorc1 Signaling Regulates Acly to Integrate Metabolic Input to Control of Macrophage Activation. elife (2016) 5:e11612. doi: 10.7554/eLife.11612

142. Park JE, Dutta B, Tse SW, Gupta N, Tan CF, Low JK, et al. Hypoxia-Induced Tumor Exosomes Promote M2-Like Macrophage Polarization of Infiltrating Myeloid Cells and microRNA-Mediated Metabolic Shift. Oncogene (2019) 26:5158-73. doi: 10.1038/s41388-019-0782-x

143. Wang W, Liu Y, Guo J, He H, Mi X, Chen C, et al. miR-100 Maintains Phenotype of Tumor-Associated Macrophages by Targeting mTOR to Promote Tumor Metastasis via Stat5a/IL-1ra Pathway in Mouse Breast Cancer. Oncogenesis (2018) 7:97. doi: 10.1038/s41389-018-0106-y

144. Yang Y, Alderman C, Sehlaoui A, Xiao Y, Wang W. MicroRNAs as Immunotherapy Targets for Treating Gastroenterological Cancers. Can J Gastroenterol Hepatol (2018) 2018:9740357. doi: 10.1155/2018/9740357

145. Wenes M, Shang M, Di Matteo M, Goveia J, Martín-Pérez R, Serneels J, et al. Macrophage Metabolism Controls Tumor Blood Vessel Morphogenesis and Metastasis. Cell Metab (2016) 24:701-15. doi: 10.1016/j.cmet.2016.09.008

146. Deng Y, Yang J, Luo F, Qian J, Liu R, Zhang D, et al. mTOR-Mediated Glycolysis Contributes to the Enhanced Suppressive Function of Murine Tumor-Infiltrating Monocytic Myeloid-Derived Suppressor Cells. Cancer Immunol Immunother (2018) 67:1355-64. doi: 10.1007/s00262-018-2177-1

147. Tesi R. MDSC; the Most Important Cell You Have Never Heard of. Trends Pharmacol Sci (2019) 40:4-7. doi: 10.1016/j.tips.2018.10.008

148. Wu T, Zhao Y, Wang H, Li Y, Shao L, Wang R, et al. mTOR Masters Monocytic Myeloid-Derived Suppressor Cells in Mice With Allografts or Tumors. Sci Rep (2016) 6:20250. doi: 10.1038/srep20250

149. Welte T, Kim IS, Tian L, Gao X, Wang H, Li J, et al. Oncogenic mTOR Signalling Recruits Myeloid-Derived Suppressor Cells to Promote Tumour Initiation. Nat Cell Biol (2016) 18:632-44. doi: 10.1038/ncb3355

150. Ryzhov SV, Pickup MW, Chytil A, Gorska AE, Zhang Q, Owens P, et al. Role of TGF- $\beta$ Signaling in Generation of CD39+ CD73+ Myeloid Cells in Tumors. J Immunol (2014) 193:3155-64. doi: 10.4049/jimmunol.1400578

151. Li J, Wang L, Chen X, Li L, Li Y, Ping Y, et al. CD39/CD73 Upregulation on Myeloid-Derived Suppressor Cells via TGF-beta-mTOR-HIF-1 Signaling in Patients With Non-Small Cell Lung Cancer. Oncoimmunology (2017) 6: e1320011. doi: 10.1080/2162402X.2017.1320011

152. Raber PL, Sierra RA, Thevenot PT, Shuzhong Z, Wyczechowska DD, Kumai $\mathrm{T}$, et al. T Cells Conditioned With MDSC Show an Increased Anti-Tumor Activity After Adoptive T Cell Based Immunotherapy. Oncotarget (2016) 7:17565-78. doi: 10.18632/oncotarget.8197

153. Mei S, Xin J, Liu Y, Zhang Y, Liang X, Su X, et al. MicroRNA-200c Promotes Suppressive Potential of Myeloid-Derived Suppressor Cells by Modulating PTEN and FOG2 Expression. PloS One (2015) 10:e0135867. doi: 10.1371/ journal.pone.0135867 
154. Shaul ME, Fridlender ZG. Tumour-Associated Neutrophils in Patients With Cancer. Nat Rev Clin Oncol (2019) 16:601-20. doi: 10.1038/s41571-019-0222-4

155. Liu L, Das S, Losert W, Parent CA. Mtorc2 Regulates Neutrophil Chemotaxis in a cAMP-And RhoA-Dependent Fashion. Dev Cell (2010) 19:845-57. doi: 10.1016/j.devcel.2010.11.004

156. Shrivastava R, Asif M, Singh V, Dubey P, Malik SA, Tewari BN, et al. M2 Polarization of Macrophages by Oncostatin $M$ in Hypoxic Tumor Microenvironment is Mediated by Mtorc2 and Promotes Tumor Growth and Metastasis. Cytokine (2019) 118:130-43. doi: 10.1016/j.cyto.2018.03.032

157. Li X-F, Chen D-P, Ouyang F-Z, Chen M-M, Wu Y, Kuang D-M, et al. Increased Autophagy Sustains the Survival and Pro-Tumourigenic Effects of Neutrophils in Human Hepatocellular Carcinoma. J Hepatol (2015) 62:1319. doi: 10.1016/j.jhep.2014.08.023

158. Wellenstein MD, De Visser KE. Cancer-Cell-Intrinsic Mechanisms Shaping the Tumor Immune Landscape. Immunity (2018) 48:399-416. doi: 10.1016/ j.immuni.2018.03.004

159. Gomez-Cambronero J. Rapamycin Inhibits GM-CSF-Induced Neutrophil Migration. FEBS Lett (2003) 550:94-100. doi: 10.1016/S0014-5793(03)00828-7

160. Chen YL, Lin HW, Sun NY, Yie JC, Hung HC, Chen CA, et al. mTOR Inhibitors Can Enhance the Anti-Tumor Effects of DNA Vaccines Through Modulating Dendritic Cell Function in the Tumor Microenvironment. Cancers (Basel) (2019) 11:617-36. doi: 10.3390/cancers11050617

161. Raich-Regue D, Fabian KP, Watson AR, Fecek RJ, Storkus WJ, Thomson AW. Intratumoral Delivery of Mtorc2-Deficient Dendritic Cells Inhibits B16 Melanoma Growth by Promoting CD8(+) Effector T Cell Responses. Oncoimmunology (2016) 5:e1146841. doi: 10.1080/2162402X.2016.1146841

162. Amiel E, Everts B, Freitas TC, King IL, Curtis JD, Pearce EL, et al. Inhibition of Mechanistic Target of Rapamycin Promotes Dendritic Cell Activation and Enhances Therapeutic Autologous Vaccination in Mice. J Immunol (2012) 189:2151-8. doi: 10.4049/jimmunol.1103741

163. Marcais A, Cherfils-Vicini J, Viant C, Degouve S, Viel S, Fenis A, et al. The Metabolic Checkpoint Kinase mTOR is Essential for IL-15 Signaling During the Development and Activation of NK Cells. Nat Immunol (2014) 15:74957. doi: 10.1038/ni.2936

164. Viel S, Marcais A, Guimaraes FS, Loftus R, Rabilloud J, Grau M, et al. TGFBeta Inhibits the Activation and Functions of NK Cells by Repressing the mTOR Pathway. Sci Signal (2016) 9:ra19. doi: 10.1126/scisignal.aad1884

165. Balsamo M, Manzini C, Pietra G, Raggi F, Blengio F, Mingari MC, et al. Hypoxia Downregulates the Expression of Activating Receptors Involved in NK-Cell-Mediated Target Cell Killing Without Affecting ADCC. Eur J Immunol (2013) 43:2756-64. doi: 10.1002/eji.201343448

166. Steggerda SM, Bennett MK, Chen J, Emberley E, Huang T, Janes JR, et al. Inhibition of Arginase by CB-1158 Blocks Myeloid Cell-Mediated Immune
Suppression in the Tumor Microenvironment. J Immunother Cancer (2017) 5:101. doi: 10.1186/s40425-017-0308-4

167. Guo S, Huang C, Han F, Chen B, Ding Y, Zhao Y, et al. Gastric Cancer Mesenchymal Stem Cells Inhibit NK Cell Function Through mTOR Signalling to Promote Tumour Growth. Stem Cells Int (2021) 2021:9989790. doi: 10.1155/2021/9989790

168. Felices M, Lenvik AJ, Mcelmurry R, Chu S, Hinderlie P, Bendzick L, et al. Continuous Treatment With IL-15 Exhausts Human NK Cells via a Metabolic Defect. JCI Insight (2018) 3:e96219. doi: 10.1172/jci.insight.96219

169. Fujii S, Shimizu K, Okamoto Y, Kunii N, Nakayama T, Motohashi S, et al NKT Cells as an Ideal Anti-Tumor Immunotherapeutic. Front Immunol (2013) 4:409. doi: 10.3389/fimmu.2013.00409

170. Gupta S, Roy A, Dwarakanath BS. Metabolic Cooperation and Competition in the Tumor Microenvironment: Implications for Therapy. Front Oncol (2017) 7:68. doi: 10.3389/fonc.2017.00068

171. Yin Z, Bai L, Li W, Zeng T, Tian H, Cui J. Targeting T Cell Metabolism in the Tumor Microenvironment: An Anti-Cancer Therapeutic Strategy. J Exp Clin Cancer Res (2019) 38:403. doi: 10.1186/s13046-019-1409-3

172. Xie D, Zhu S, Bai L. Lactic Acid in Tumor Microenvironments Causes Dysfunction of NKT Cells by Interfering With mTOR Signaling. Sci China Life Sci (2016) 59:1290-6. doi: 10.1007/s11427-016-0348-7

173. Shin J, Wang S, Deng W, Wu J, Gao J, Zhong X-P. Mechanistic Target of Rapamycin Complex 1 Is Critical for Invariant Natural Killer T-Cell Development and Effector Function. PNAS (2014) 111:E776-83. doi: $10.1073 /$ pnas. 1315435111

Conflict of Interest: The authors declare that the research was conducted in the absence of any commercial or financial relationships that could be construed as a potential conflict of interest.

Publisher's Note: All claims expressed in this article are solely those of the authors and do not necessarily represent those of their affiliated organizations, or those of the publisher, the editors and the reviewers. Any product that may be evaluated in this article, or claim that may be made by its manufacturer, is not guaranteed or endorsed by the publisher.

Copyright (C) 2022 Mafi, Mansoori, Taeb, Sadeghi, Abbasi, Cho and Rostamzadeh. This is an open-access article distributed under the terms of the Creative Commons Attribution License (CC BY). The use, distribution or reproduction in other forums is permitted, provided the original author(s) and the copyright owner(s) are credited and that the original publication in this journal is cited, in accordance with accepted academic practice. No use, distribution or reproduction is permitted which does not comply with these terms. 